\title{
A Comparative Study on the Solution Techniques for Fiber Orientation in Two-Dimensional Converging and Diverging Flows
}

\author{
B. N. Rao, S. Akbar And M. C. Altan \\ School of Aerospace and Mechanical Engineering \\ University of Oklahoma \\ Norman, OK 73019
}

\begin{abstract}
The two-dimensional steady flow of both infinite and finite aspect ratio (length to diameter ratio) fibers suspended in a Newtonian fluid is investigated numerically. Forty-five-degree convergent and divergent channel geometries are considered for the analysis. Due to symmetry, only half the channel geometry is considered and the orientation field is assumed to be planar. The analysis is carried out for the creeping flows where the inertia terms are neglected. Numerical grid generation is used to generate the mesh, and the transformed governing equations in terms of the stream function are solved in the computational domain using a finite difference scheme. In this study, several solution strategies for solving the orientation field are investigated. The orientation of individual fibers are assumed to be governed by Jeffery's equation. The orientation field, which can be expressed in different forms (i.e., a unit vector, tensorial quantities, or an orientation distribution function), is specified by solving the orientation equations along particle paths. A tracing technique is implemented to obtain these particle paths for each grid point in the flow domain. The solution of the orientation field is obtained by using two basic techniques. First, a large number of fibers are considered, and by using analytical expressions developed to describe the orientation state of one fiber, a statistical orientation distribution function is generated. Second, tensorial quantities (both second- and fourth-order orientation tensors) are employed to solve for the orientation field. In order to overcome the closure problem occurring in the resulting orientation equations, quadratic approximations are used. Maximum orientation angles are reported from both the techniques, and their accuracies are investigated. The maximum orientation angles (i.e., preferred orientation) obtained from the second- and fourth-order tensorial solutions are observed to be identical. On the other hand, the degree of fiber alignments that are specified by the individual tensor components differ considerably. Comparison of the solution techniques shows that the accuracy of the preferred angle obtained from statistical solution is dependent on the number of fibers considered. In addition, the calculations for the finite fiber aspect ratio revealed some discrepancies between the statistical and tensorial results at the regions of rapid fiber tumbling.
\end{abstract}




\section{INTRODUCTION}

7 THE MECHANICAL PROPERTIES of short fiber composites are highly dependent 1 on microstructural characteristics such as the fiber volume concentration, fiber geometry, and the orientation distribution of the fibers. The fiber orientation structure in these materials, apart from being affected by volume concentration and aspect ratio of the fibers, is influenced by the processing conditions used in their manufacture. Experimental evidence has shown that reinforced thermoplastics have the highest strength in the preferred orientation direction of the fibers. Although the strength characteristics of these composites can be determined from micro-mechanical constitutive models, in order to use these models, a prior knowledge of the fiber orientation field is necessary. Thus, if the fiber orientation can be predicted for a given set of processing conditions, manufacturing short fiber composites with the optimum mechanical properties will become possible.

Jeffery [1], in 1922, studied the motion of an ellipsoidal particle immersed in a homogeneous flow of a Newtonian fluid, and developed an expression describing the particle motion and the forces acting on the suspended particle. Since then, several constitutive models [2-6] have been developed from which the flow induced orientation can be predicted for the dilute or semiconcentrated suspensions. In general, these constitutive models treat the suspension as a continuum and express the bulk stresses generated in the suspensions as:

$$
\begin{gathered}
\sigma_{i j}=\sigma_{i j}^{f}+\sigma_{i j}^{P} \\
\sigma_{i j}^{f}=\mu\left(u_{i j}+u_{j i}\right) \\
\sigma_{i j}^{p}=\mu \phi_{v}\left(A S_{i j k l} u_{k l}+B\left[S_{i k} u_{k j}+u_{i k} S_{k j}\right]+C u_{i j}\right)
\end{gathered}
$$

where $\sigma_{i j}^{f}$ and $\sigma_{i j}^{p}$ are the stress contributions from the suspending fluid and the particles respectively, $\mu$ is the viscosity of the suspending fluid, $u_{i j}$ is the velocity gradient tensor, $\phi_{v}$ is the fiber volume fraction, $S_{i j}$ and $S_{i j k l}$ are the second- and fourth-order tensors which account for the orientation distribution of fibers, and $A, B, C$ are the material constants that depend on the particle geometry. A detailed discussion on various constitutive models and equations for the material constants can be found in Tucker's recent publication [7].

In a complex flow, if the spatial stress gradients due to fibers are very small compared to spatial viscous stress gradients, then the fluid behavior is Newtonian (i.e., the presence of fibers does not alter the flow kinematics). Consequently, the implementation of an anisotropic model is not needed and the sole use of Jeffery's equation is sufficient to characterize the orientation field. As the expression of $\sigma_{i j}^{p}$ indicates, the particle stress contribution linearly varies with the fiber volume fraction $\phi_{v}$ and therefore, in any flow there exists a critical particle volume fraction below which the suspension can be regarded as Newtonian. For such cases, Givler et al. [8] solved the planar fiber orientation field of complex twodimensional flows by numerically integrating Jeffery's orientation equation along 
the streamlines. On the other hand, if stress gradient contributions from the particles are comparable or larger than the suspending fluid contributions, the suspension exhibits non-Newtonian characteristics with directional dependent properties (i.e., anisotropic) which necessitates the simultaneous solution of the flow and orientation fields by using a proper constitutive model.

A few numerical solutions to the above described coupled problem (i.e., accounting the affect of particles on the flow field by utilizing an anisotropic constitutive model) have recently been reported. Papanastasiou and Alexandrou [9] worked on the isothermal extrusion of nondilute fiber suspensions. They utilized Dinh-Armstrong constitutive equations and resulting nonlinear integrodifferential equations were solved with a streamlined finite element technique. Lipscomb et al. [5] utilized a similar constitutive equation proposed by Evans [3], and solved the orientation field in a sudden contraction using a finite element method. Later, Chiba et al. [10], using a finite difference technique, confirmed and extended Lipscomb's work considering the effects of fluid inertia. Rosenberg et al. [11] investigated nonrecirculating flows of fiber suspensions and reported results for falling-ball rheometry. Phan-Thien and Graham [6] proposed a new constitutive model that suggests a new functional form for the material coefficient $A$ shown in Equation (1). In their work, flow past a sphere is investigated and comparisons with experimental data showed good agreement. Altan et al. [12] obtained numerical solutions of planar fiber orientation in a straight channel using Dinh-Armstrong's constitutive equations. In this work a finite difference technique is used and the effect of fibers on the flow profile is investigated. Apart from the numerical solutions, researchers have also carried out experiments which have served as a suitable measure for comparison with the numerical results. Goldsmith and Mason [13] performed a series of experiments to investigate the orientation of rigid ellipsoids and the stresses acting on the particles in shear flow. Vincent and Agassant [14] investigated orientation of short fibers (copper and glass) suspended in corn syrup in diverging flows. Lipscomb et al. [5] conducted experiments on the flow of chopped-glass fiber suspensions through axisymmetric contractions and found excellent agreement with the computed results.

In processing of polymers, flows are characterized by low Reynolds numbers, where the viscous effects are dominant compared to the inertial effects. For such cases, the creeping flow equations become the governing equations of flow. For the simultaneous solution of the flow and orientation fields, first, it is convenient to compute the orientation of the fibers assuming that the stresses generated due to presence of fibers are zero. Then, the orientation field computed from the Newtonian solution is coupled back to the governing equations of flow to solve the anisotropic flow of fiber suspensions. In this work, the orientation field, which can be expressed in different forms (i.e., a unit vector, tensorial quantities, or an orientation distribution function), is obtained by solving the orientation equations along particle streamlines. Along these particle paths, average velocity gradients and the time periods during which these velocity gradients are effective are used to calculate the orientation of fibers. It is necessary that small intervals be used to compute the orientation field, because the orientation of the fibers is 
affected only by the local velocity gradients. Our aim in this paper is to present and investigate the accuracies of the various solution techniques for the solution of orientation field in a Newtonian fluid where the particles do not alter the flow kinematics. For suspensions with higher volume fractions, these solution techniques can be utilized in conjunction with the anisotropic constitutive models for the accurate representation of the flow behavior.

In this paper, the two-dimensional steady flow of both infinite and finite aspect ratio fibers suspended in a Newtonian fluid is investigated numerically. Since the effect of the fibers on the velocity field is neglected, the orientation results are valid below a critical fiber volume concentration where the suspension exhibits Newtonian flow characteristics. Forty-five-degree convergent and divergent channel geometries are considered for the study. The analysis is carried out for creeping flows where the inertia terms are neglected. In order to describe orientation field (assumed to be planar) throughout the flow domain, several solution strategies and their accuracies are investigated. Two basic techniques are considered for the solution of the orientation field. First, a large number of fibers, each starting from different initial orientation are considered. By suitably choosing the initial orientation angles of individual fibers, it is possible to impose a meaningful initial orientation condition, ranging from random to complete alignment. Starting from the specified initial orientations, the orientation vector for each fiber is solved along the streamlines generating a statistical distribution function. In these solutions, analytical expressions developed by Akbar and Altan [15] are used and the maximum orientation angles (preferred orientation) throughout the converging and diverging channels are reported. Second, tensorial quantities are used to solve the orientation field. The orientation evolution equations for second- and fourth-order orientation tensors are employed. Similarly, the maximum orientation angle is computed from the second- and fourth-order orientation tensor components. For the flow geometries, the orientation angle results obtained using the second-order tensors, fourth-order tensors, and the statistical solution utilizing the orientation distribution of the numerous fibers are compared. The orientation angles obtained from the second- and fourth-order solutions are identical whereas the degree of fiber alignments differ considerably. Comparison with the statistical results show that the accuracy of the statistical solution is dependent on the number of fibers considered. Satisfactory results are obtained for ninety fibers in the statistical solution. However, considering only nine fibers revealed slight errors in the orientation predictions. Hence, depending on the available computational resources and desired accuracy level, one can prefer to trace different numbers of particles for the simulation of fiber suspensions.

\section{THEORY}

\section{Description of Orientation State}

\section{ORIENTATION VECTOR}

The orientation vector $\vec{p}$ is defined as a unit vector which represents the orien- 
tation of a single fiber. This unit vector is taken to coincide with the axis of revolution of the fiber geometry.

The rotation of a rigid fiber with the bulk fluid motion can be expressed in terms of orientation vector components. In the case of planar (2-D) representation of fiber orientation, the orientation vector components $p_{1}$ and $p_{2}$ are related to the orientation angle $\phi$ as

$$
\vec{p}=\left\{\begin{array}{l}
p_{1} \\
p_{2}
\end{array}\right\}=\left(\begin{array}{c}
\cos \phi \\
\sin \phi
\end{array}\right\}
$$

where, the angle $\phi$ is measured from the $x$ axis. Therefore any change in the orientation angle would manifest itself in a change of the orientation vector components or vice-versa. This was shown by Jeffery's equation for the motion of an ellipsoid of revolution immersed in a viscous medium as

$$
\dot{p}_{i}=\left(\Omega_{i j}+\lambda \Delta_{i j}\right) p_{j}-\lambda p_{i} p_{l} p_{k} \Delta_{l k}
$$

where $\dot{p}_{i}$ represents the time rate of change in the orientation vector components, and $\Omega_{i j}$ and $\Delta_{i j}$ are the vorticity and strain rate tensors respectively.

$$
\begin{aligned}
& \Omega_{i j}=\frac{1}{2}\left(\frac{\partial u_{i}}{\partial x_{j}}-\frac{\partial u_{j}}{\partial x_{i}}\right) \\
& \Delta_{i j}=\frac{1}{2}\left(\frac{\partial u_{i}}{\partial x_{j}}+\frac{\partial u_{j}}{\partial x_{i}}\right)
\end{aligned}
$$

In the above equations, $i, j=1,2$, and summation is implied over repeated indices. The parameter $\lambda$ is a function of the fiber aspect ratio $a_{p}$, and is given as

$$
\lambda=\frac{a_{p}^{2}-1}{a_{p}^{2}+1}
$$

Equation (3) takes into account the effect of flow kinematics on a rigid inextensible fiber, which is unable to follow the bulk fluid rotation affinely.

The solution to Jeffery's equation with the initial condition $p_{l}=p_{i}^{o}$ has been shown in Reference [4] to be

$$
p_{i}=\frac{E_{i l} p_{i}^{o}}{\left(E_{l m} E_{l j} p_{j}^{o} p_{m}^{o}\right)^{1 / 2}}
$$

where $E_{i j}$ 's are the components of a strain tensor defined as

$$
\frac{d E_{i j}}{d t}=\left(\Omega_{i k}+\lambda \Delta_{i k}\right) E_{k j}
$$


Equations (6) and (7) constitute a simple solution to the Jeffery's equation where the fiber is subjected to a homogeneous flow field characterized by $\Omega_{i j}$ and $\Delta_{i j}$. This solution is valid for both two- and three-dimensional flows and orientation fields. For infinite aspect ratio fibers (slender fibers) $\lambda=1$, and Equation (7) reduces to

$$
E_{i j}=\frac{\partial x_{i}}{\partial x_{j}^{\circ}}
$$

where $x_{i}$ and $x_{i}^{o}$ are the fluid particle coordinates at times $t$ and $t^{\circ}$ respectively.

Hence, the orientation vector components can be easily calculated from the flow kinematics and initial fiber orientation using Equation (6). To describe a rheologically meaningful orientation state, same analysis can be used for numerous fibers in the domain, generating a statistical distribution function. However, this representation may lead to an inaccurate description of the orientation state if the number of fibers considered are not high enough.

\section{DISTRIBUTION FUNCTION}

The orientation distribution function $\Psi(\vec{p}, t)$ provides a complete and accurate representation of the fiber orientation state. It gives the probability of having a fiber at a certain orientation $\vec{p}$ at any time $t$. Equivalently, it can also be defined in terms of the orientation angle as the probability of finding a fiber in a certain angular interval between $\phi_{1}$ and $\phi_{2}$ at any time $t$.

For two-dimensional (planar) representation of fiber orientation state, the orientation distribution function should satisfy certain conditions. Since one end of a fiber is not distinguishable from the other, the distribution function has a period of $\pi$, or

$$
\Psi(\phi)=\Psi(\phi+\pi)
$$

It also satisfies the normalization condition

$$
\int_{0}^{\pi} \Psi(\phi) d \phi=1
$$

which implies that the area under a distribution function curve is always unity.

An explicit equation for the orientation distribution function is obtained by solving the governing equation for distribution function given as

$$
\frac{\partial \Psi(\vec{p}, t)}{\partial t}=-\frac{\partial\left[\dot{p}_{i} \Psi(\vec{p}, t)\right]}{\partial p_{i}}
$$

where $i=1,2$ and $\dot{p}_{i}$ is given by Equation (3). The governing equation for orientation distribution function is a form of the Fokker-Planck equation used for 
homogeneous flows with negligible Brownian motion. For planar orientations, the analytical solution to Equation (11) can be expressed as

$$
\Psi(\vec{p}, t)=\frac{1}{\pi}\left(\Lambda_{l m} \Lambda_{l j} p_{j} p_{m}\right)^{-1}
$$

where $\Lambda_{i j}$ is called the deformation gradient tensor and is the inverse of the strain tensor $E_{i j}$. The analytical solution for orientation distribution function given in Equation (12) is only valid for random initial orientation of fibers. Using this initial condition, the normalization condition is obtained as

$$
\Psi(\phi, t=0)=\frac{1}{\pi}
$$

Equation (12) has been used in Reference [16] to evaluate the orientation distribution function for various two- and three-dimensional flow fields for infinite aspect ratio fibers.

The analytical solution of the Fokker-Planck equation as given in Equation (12) is not available for nonrandom initial orientations. This severely restricts the applicability of the distribution function for complex flows where the solution of the orientation state along a streamline is required.

\section{ORIENTATION TENSORS}

Although the statistical orientation distribution function provides a complete and accurate description of the orientation state, it has been shown that the orientation state of fibers in a suspension can be described with reasonable accuracy with tensors [17]. A number of researchers [5,6,12] have used these tensors in the numerical computation of the orientation of fibers successfully. Even though the accuracy of the orientation description improves when a higher order tensor is used [17], second- and fourth-order tensors were found to be adequate for the present analysis. The second- and the fourth-order (i.e., $S_{i j}$ and $S_{i j k l}$, respectively) orientation tensors are defined as

$$
\begin{aligned}
S_{i j} & =\left\langle p_{i} p_{j}\right\rangle=\int p_{i} p_{j} \Psi(\vec{p}) d \vec{p} \\
S_{i j k l} & =\left\langle p_{i} p_{j} p_{k} p_{l}\right\rangle=\int p_{i} p_{j} p_{k} p_{l} \Psi(\vec{p}) d \vec{p}
\end{aligned}
$$

The order of the indices is not important since the orientation tensors are completely symmetric. An important property of orientation tensors is that the higher order tensors contain the lower order ones. Thus, the lower order orientation tensors can be written in terms of the higher order orientation tensors. For example, any second-order tensor component can be expressed in terms of fourth-order components as

$$
S_{i j}=S_{i j k k}
$$


In addition, the trace of the second-order tensor is unity

$$
S_{i i}=1
$$

The eigenvectors and eigenvalues of the second-order tensor indicate the principal orientation direction of the fiber and the degree of alignment in that direction. The magnitude of the preferred orientation angle is computed from the components of the second-order orientation tensor.

\section{Governing Equations}

\section{FLOW EQUATIONS}

Since the flow is assumed to be steady and the inertia terms are neglected in this analysis, the flow is governed by the biharmonic equation. In Cartesian coordinates $(x, y)$, the governing equation can be written as

$$
\frac{\partial^{4} \psi}{\partial x^{4}}+\frac{\partial^{4} \psi}{\partial y^{4}}+2 \frac{\partial^{4} \psi}{\partial x^{2} \partial y^{2}}=0
$$

where $\psi$ is the stream function defined by $u=\partial \psi / \partial y ; v=-\partial \psi / \partial x$. Here, $u$ and $v$ are the components of the velocity vector in the $x$ and $y$ direction respectively. For arbitrary shaped geometries, boundary conditions become difficult to implement if a regular Cartesian grid is used. To overcome this difficulty, a grid generation technique is used to transform the above equation to a computational space $(\xi, \eta)$ and solve the transformed equation in the computational domain. The reader is referred to Reference [18] for a complete treatment on numerical grid generation. After transformation, the governing equation becomes

$$
\begin{aligned}
\xi_{x}^{2} \frac{\partial^{2} P}{\partial \xi^{2}} & +2 \xi_{x} \eta_{x} \frac{\partial^{2} P}{\partial \xi \partial \eta}+\eta_{x}^{2} \frac{\partial^{2} P}{\partial \eta^{2}}+\xi_{x x} \frac{\partial P}{\partial \xi}+\eta_{x x} \frac{\partial P}{\partial \eta}+\xi_{y}^{2} \frac{\partial^{2} Q}{\partial \xi^{2}} \\
& +2 \xi_{y} \eta_{y} \frac{\partial^{2} Q}{\partial \xi \partial \eta}+\eta_{y}^{2} \frac{\partial^{2} Q}{\partial \eta^{2}}+\xi_{y y} \frac{\partial Q}{\partial \xi}+\eta_{y y} \frac{\partial Q}{\partial \eta}+2\left[\xi_{y} \xi_{x} \frac{\partial^{2} R}{\partial \xi^{2}}\right. \\
& \left.+\left(\xi_{y} \eta_{x}+\eta_{y} \xi_{x}\right) \frac{\partial^{2} P}{\partial \xi \partial \eta}+\eta_{y} \eta_{x} \frac{\partial^{2} R}{\partial \eta^{2}}+\xi_{y x} \frac{\partial R}{\partial \xi}+\eta_{y x} \frac{\partial R}{\partial \eta}\right]=0
\end{aligned}
$$

where

$$
\begin{aligned}
P & =\frac{\partial^{2} \psi}{\partial x^{2}}=\xi^{2} \frac{\partial^{2} \psi}{\partial \xi^{2}}+2 \xi_{x} \eta_{x} \frac{\partial^{2} \psi}{\partial \xi \partial \eta}+\eta_{x}^{2} \frac{\partial^{2} \psi}{\partial \eta^{2}} \\
& +\xi_{x x} \frac{\partial \psi}{\partial \xi}+\eta_{x x} \frac{\partial \psi}{\partial \eta}
\end{aligned}
$$




$$
\begin{aligned}
Q & =\frac{\partial^{2} \psi}{\partial y^{2}}=\xi_{y}^{2} \frac{\partial^{2} \psi}{\partial \xi^{2}}+2 \xi_{y} \eta_{y} \frac{\partial^{2} \psi}{\partial \xi \partial \eta}+\eta_{y}^{2} \frac{\partial^{2} \psi}{\partial \eta^{2}} \\
& +\xi_{y y} \frac{\partial \psi}{\partial \xi}+\eta_{y y} \frac{\partial \psi}{\partial \eta} \\
R & =\frac{\partial^{2} \psi}{\partial x \partial y}=\xi_{y}\left[\xi_{x} \frac{\partial^{2} \psi}{\partial \xi^{2}}+\eta_{x} \frac{\partial^{2} \psi}{\partial \xi \partial \eta}\right]+\xi_{y x} \frac{\partial \psi}{\partial \xi} \\
& +\eta_{y}\left[\xi_{x} \frac{\partial^{2} \psi}{\partial \xi \partial \eta}+\eta_{x} \frac{\partial^{2} \psi}{\partial \eta^{2}}\right]+\eta_{y x} \frac{\partial \psi}{\partial \eta}
\end{aligned}
$$

The metrics $\xi_{x}, \eta_{x}$ etc. are defined in the Appendix. Clearly, these metrics are functions of the generated grid and are evaluated numerically. The governing equation given in Equation (18) is discretized using a finite difference scheme and solved for the stream function in a computational plane $\xi-\eta$.

\section{ORIENTATION EQUATIONS}

As stated earlier, most constitutive models assume that the orientation of individual fibers is governed by the Jeffery's equation given by Equation (3). In order to obtain the orientation equations, the definitions of the second- and fourth-order orientation tensors are used as a starting point. Taking the derivative of Equation (14) with respect to time and using Equation (3), the second- and the fourth-order orientation evolution equations can be written with the indicial notion:

$$
\begin{aligned}
\frac{d S_{i j}}{d t} & =\Omega_{i m} S_{m j}+\Omega_{j m} S_{m i}+\lambda\left[\Delta_{i m} S_{m j}+\Delta_{j m} S_{m i}-2 \Delta_{k l} S_{i j k l}\right] \\
\frac{d S_{i j k l}}{d t} & =\left(\Omega_{i m}+\lambda \Delta_{i m}\right) S_{m j k l}+\left(\Omega_{j m}+\lambda \Delta_{j m}\right) S_{m i k l} \\
& +\left(\Omega_{k m}+\lambda \Delta_{k m}\right) S_{m i j l}+\left(\Omega_{l m}+\lambda \Delta_{l m}\right) S_{m i j k}-4 \lambda \Delta_{r s} S_{i j k l r s}
\end{aligned}
$$

The components of the orientation tensors are obtained from the solution of the resulting orientation equations. Since calculation of the orientation tensors only requires solving the above ordinary differential equations, the tedious computation of the orientation distribution function is totally avoided. It can easily be seen that three orientation equations result from Equation (22) and five from Equation (23). Although, three and five equations result from Equations (22) and (23) respectively, using the property $S_{i i}=1$, the number of independent components can be reduced to two in the case of a second-order tensor and to four in the case of a fourth-order tensor. However, these equations cannot be solved readily due to the unknowns appearing in the form of higher order tensors. Specifically, Equation (22) contains the unknown fourth-order orientation tensor 
$S_{i j k l}$, while Equation (23) contains the unknown sixth-order orientation tensor $S_{i j k l r s}$. In order to solve this problem, a quadratic closure approximation is used. With the help of these approximations, the unknowns are reduced in terms of lower order tensors:

$$
\begin{gathered}
S_{i j k l} \cong S_{i j} S_{k l} \\
S_{i j k l r s} \cong S_{i j k l} S_{r s}
\end{gathered}
$$

Altan [12] has successfully used quadratic closure approximations to solve the anisotropic flow of fiber suspensions in a two-dimensional straight channel. Lipscomb [5] has also used the same approximation in order to obtain solutions for the flow of dilute suspensions in a sudden contraction. By means of the quadratic closure approximation and the properties of orientation tensors defined earlier the second-order evolution equation becomes

$$
\begin{aligned}
\frac{d S_{11}}{d t} & =2\left[\left(\Omega_{11}+\lambda \Delta_{11}\right) S_{11}+\left(\Omega_{12}+\lambda \Delta_{12}\right) S_{21}\right. \\
& \left.-\lambda\left\{\Delta_{11} S_{11}+2 \Delta_{12} S_{12}+\Delta_{22} S_{22}\right\} S_{11}\right] \\
\frac{d S_{12}}{d t} & =\left(\Omega_{21}+\lambda \Delta_{21}\right) S_{11}+\left(\Omega_{12}+\lambda \Delta_{12}\right) S_{22} \\
& -2 \lambda\left[\left(\Delta_{11} S_{11}+2 \Delta_{12} S_{12}+\Delta_{22} S_{22}\right) S_{12}\right] \\
\frac{d S_{22}}{d t} & =2\left[\left(\Omega_{21}+\lambda \Delta_{21}\right) S_{12}+\left(\Omega_{22}+\lambda \Delta_{22}\right) S_{22}\right. \\
& \left.-\lambda\left\{\Delta_{11} S_{11}+2 \Delta_{12} S_{12}+\Delta_{22} S_{22}\right\} S_{22}\right]
\end{aligned}
$$

Also, the resulting evolution equations for the fourth-order orientation tensor are given by

$$
\begin{aligned}
\frac{d S_{1111}}{d t}= & 4\left[\left(\Omega_{11}+\lambda \Delta_{11}\right) S_{1111}+\left(\Omega_{12}+\lambda \Delta_{12}\right) S_{1112}\right. \\
- & \lambda\left\{\Delta_{11} S_{1111}+\left(\Delta_{11}+\Delta_{22}\right) S_{1122}+\left(\Delta_{12}+\Delta_{21}\right)\right. \\
& \left.\left.\left(S_{1112}+S_{1222}\right)+\Delta_{22} S_{2222}\right\} S_{1111}\right] \\
\frac{d S_{1112}}{d t}= & 3\left[\left(\Omega_{11}+\lambda \Delta_{11}\right) S_{1112}+\left(\Omega_{12}+\lambda \Delta_{12}\right) S_{1122}\right] \\
+ & \left(\Omega_{21}+\lambda \Delta_{21}\right) S_{1111}+\left(\Omega_{22}+\lambda \Delta_{22}\right) S_{1112} \\
- & 4 \lambda\left[\left(\Delta_{11} S_{1111}+\left(\Delta_{11}+\Delta_{22}\right) S_{1122}\right.\right. \\
+ & \left.\left.\left(\Delta_{12}+\Delta_{21}\right)\left(S_{1112}+S_{1222}\right)+\Delta_{22} S_{2222}\right\} S_{1112}\right]
\end{aligned}
$$




$$
\begin{aligned}
\frac{d S_{1122}}{d t}= & 2\left[\left(\Omega_{11}+\lambda \Delta_{11}\right) S_{1122}+\left(\Omega_{12}+\lambda \Delta_{12}\right) S_{1222}\right] \\
& +\left(\Omega_{21}+\lambda \Delta_{21}\right) S_{1112}+\left(\Omega_{22}+\lambda \Delta_{22}\right) S_{1122} \\
& -4 \lambda\left[\left\{\Delta_{11} S_{1111}+\left(\Delta_{11}+\Delta_{22}\right) S_{1122}\right.\right. \\
+ & \left.\left.\left(\Delta_{12}+\Delta_{21}\right)\left(S_{1112}+S_{1222}\right)+\Delta_{22} S_{2222}\right\} S_{1122}\right] \\
\frac{d S_{1222}}{d t}= & \left(\Omega_{11}+\lambda \Delta_{11}\right) S_{1222}+\left(\Omega_{12}+\lambda \Delta_{12}\right) S_{2222} \\
+ & 3\left[\left(\Omega_{21}+\lambda \Delta_{21}\right) S_{1122}+\left(\Omega_{22}+\lambda \Delta_{22}\right) S_{1222}\right] \\
- & 4 \lambda\left[\left\{\Delta_{11} S_{1111}+\left(\Delta_{11}+\Delta_{22}\right) S_{1122}\right.\right. \\
+ & \left.\left.\left(\Delta_{12}+\Delta_{21}\right)\left(S_{1112}+S_{1222}\right)+\Delta_{22} S_{2222}\right\} S_{1222}\right] \\
\frac{d S_{2222}}{d t}= & 4\left[\left(\Omega_{21}+\lambda \Delta_{21}\right) S_{1222}+\left(\Omega_{22}+\lambda \Delta_{22}\right) S_{2222}\right. \\
- & \lambda\left\{\Delta_{11} S_{1111}+\left(\Delta_{11}+\Delta_{22}\right) S_{1122}+\left(\Delta_{12}+\Delta_{21}\right)\right. \\
& \left.\left.\left(S_{1112}+S_{1222}\right)+\Delta_{22} S_{2222}\right\} S_{2222}\right]
\end{aligned}
$$

The second- and the fourth-order orientation tensor components are obtained from these ordinary differential Equations (25-32) which are solved along the particle paths using the Livermore Solver for Ordinary Differential Equations (LSODE).

\section{SOLUTION TECHNIQUES}

\section{Solution Technique for Biharmonic Equation}

The transformed governing equations are solved using a finite difference scheme in the computational domain $(\xi, \eta)$. The stream function is represented explicitly which enables it to be solved iteratively using a Gauss-Seidel technique with SOR (Successive Over Relaxation) to speed up convergence. In order to solve Equation (18), a set of fictitious nodes is necessary around the domain of interest so that the boundary conditions are satisfied. The boundary conditions for the stream function calculation for both the channel geometries are the same except at the inlet of the channel. Because of symmetry, only half of the channel geometry is considered as shown in Figures 3 and 7. On the channel centerline, the velocity gradients with respect to $y$ is zero. On the channel wall, no-slip boundary condition is imposed, and at the channel exit, fully developed flow is assumed. The boundary condition at the inlet is dependent on the channel geometry. In the case of a convergent channel, radial velocity is specified at the inlet. 
The radial velocity, $v_{r}$, is given by

$$
v_{r}=\frac{Q}{r_{\varrho}}\left[\frac{\cos 2 \theta-\cos 2 \alpha}{\sin 2 \alpha-2 \alpha \cos 2 \alpha}\right]
$$

where $Q$ is the volume flow rate, $\varrho$ is the density of the fluid, $r$ and $\theta$ are variables in the radial and angular directions respectively, and $\alpha$ (forty-five degrees for the considered geometry) is the angle of convergence. In the divergent channel geometry, a parabolic velocity profile given by

$$
u=u_{\max }\left(1-y^{2}\right) \quad v=0
$$

is specified at the inlet. Here, $u_{\max }$ is the maximum velocity and is equal to unity. After the expansion, a straight channel is used long enough to ensure a fully developed velocity profile at the exit.

\section{Solution Technique for Orientation Evolution Equations}

In order to extract the orientation information in terms of the second- and fourth-order tensor components, it is necessary to solve the orientation Equations (25-32) at each nodal point in the flow domain along particle streamlines. Therefore, a tracing technique is implemented to trace the stream function value from every single nodal point in the domain back to the inlet, thus specifying individual particle paths. Along each particle path corresponding to a single nodal point, average velocity gradients and the time spent in the interval defined by adjacent mesh points are computed. This information is employed in the LSODE and the procedure is repeated, constantly updating the orientation information until the whole path is traversed up to the nodal point of interest. This procedure is not valid for the nodes on the channel wall where the velocity vector is zero, and for the nodes on the inlet of the channel, where a random initial orientation is specified using the definition of the second- and fourth-order orientation tensors. Hence, using Equation (14), the following values are obtained for the second-order orientation tensor at the inlet:

$$
\begin{aligned}
& S_{11}=S_{22}=\frac{1}{2} \\
& S_{12}=0
\end{aligned}
$$

For the fourth-order tensor components

$$
\begin{aligned}
& S_{111}=S_{2222}=\frac{3}{8} \\
& S_{1112}=S_{1222}=0
\end{aligned}
$$




$$
S_{1122}=\frac{1}{8}
$$

On the wall boundary, the orientation of the fibers is specified to be aligned with the channel wall.

After the solution of $S_{i j}$ and $S_{i j k l}$ along the particle pathlines for each node, maximum orientation angle is calculated. From second-order orientation tensor $S_{i j}$, the principal orientation directions can be calculated as:

$$
\tan 2 \phi=\frac{2 S_{12}}{S_{11}-S_{22}}
$$

Similarly, from fourth-order orientation tensor

$$
\tan 2 \phi=\frac{2\left(S_{1112}-S_{1222}\right)}{S_{111}-S_{2222}}
$$

Each one of the equations above defines two particular values of angle $2 \phi$, one of which defines the maximum orientation angle $\phi_{\max }$ and the other, the minimum orientation angle $\phi_{\min }$. In fact, $\phi_{\max }$ and $\phi_{\min }$ specify the eigenvectors of $S_{i j}$ which can be utilized to construct an orientation ellipse.

One other important parameter to analyze is the degree of fiber alignment along the maximum orientation angle $\phi_{\max }$. For the second-order tensor $S_{i j}$ the degree of alignment along $\phi_{\max }$ can be expressed as:

$$
\lambda_{1}=\frac{S_{11}+S_{22}}{2}+\sqrt{\left(\frac{S_{11}-S_{22}}{2}\right)^{2}+S_{12}^{2}}
$$

From $\lambda_{1}$ and using $\lambda_{1}+\lambda_{2}=1$, the degree of alignment, $\lambda_{2}$, along the minimum orientation angle, $\phi_{\min }$, can also be calculated.

In a similar manner, from the fourth-order orientation tensor

$\lambda_{1}=\frac{S_{1111}+2 S_{1122}+S_{2222}}{2}+\sqrt{\left(\frac{S_{1111}-S_{2222}}{2}\right)^{2}+\left(S_{1112}+S_{1222}\right)^{2}}$

For random orientation state, $\lambda_{1}=\lambda_{2}=0.5$ whereas for perfect alignment $\lambda_{1}=1 ; \lambda_{2}=0$.

In this study, only the orientation angles are depicted as orientation results. Therefore at the nodal points of the flow domains, the direction of line segments of equal length indicates the maximum orientation angle $\phi_{\max }$.

\section{Solution Technique for Discrete Fibers}

For any two-dimensional flow field the velocity gradient tensor can be specified as 


$$
u_{i j}=\left(\begin{array}{ll}
\frac{\partial u_{1}}{\partial x_{1}} & \frac{\partial u_{1}}{\partial x_{2}} \\
\frac{\partial u_{2}}{\partial x_{1}} & \frac{\partial u_{2}}{\partial x_{2}}
\end{array}\right)=\left(\begin{array}{cc}
c & c_{1} \\
c_{2} & -c
\end{array}\right)
$$

where $c, c_{1}$, and $c_{2}$ are constants, and the trace of the tensor should be zero to satisfy the conservation of mass. This velocity gradient tensor can be used to represent various kinds of homogeneous flows by changing the values of velocity gradients. Hence the flow kinematics can be altered and the subsequent effect incorporated in terms of the equivalent strain tensor.

The strain tensor evaluated from Equation (7) is utilized in Equation (6) for calculation of orientation vector components. The initial orientation vector components are related to the corresponding orientation angle, $\phi_{o}$, by

$$
\begin{aligned}
& p_{1}^{o}=\cos \phi_{o} \\
& p_{2}^{o}=\sin \phi_{o}
\end{aligned}
$$

Once the orientation vector components are known at any instant, the orientation angle, $\phi$, can be calculated as

$$
\tan \phi=\frac{p_{2}}{p_{1}}
$$

where the angle, $\phi$, is measured from the $x$ axis. Using this technique, the analytical solution of fiber orientation has been shown by solving the system of four first-order coupled differential equations obtained from Equation (7) [15].

To calculate the strain tensor in terms of velocity gradients, the four coupled differential equations have to be solved with the initial condition $\mathbf{E}=\mathbf{I}$ (unit tensor). Three categories of solutions can be identified for the system of differential equations depending on $w^{2}$ shown below, which appears as a coefficient in the differential equations

$$
w^{2}=\frac{\lambda^{2}\left(A_{1}+4 c^{2}\right)-A_{2}^{2}}{4}
$$

where $A_{1}=c_{1}+c_{2}$ and $A_{2}=c_{1}-c_{2}$. Depending on the value of $w^{2}$, the following three categories of solutions for the strain tensor are possible:

$$
\begin{aligned}
& \text { When } w^{2}=0 \\
& E_{i j}=\left(\begin{array}{cc}
c \lambda t+1 & \frac{\left(\lambda A_{1}+A_{2}\right) t}{2} \\
\frac{\left(\lambda A_{1}-A_{2}\right) t}{2} & 1-c \lambda t
\end{array}\right)
\end{aligned}
$$


When $w^{2}>0$

$$
E_{i j}=\left(\begin{array}{cc}
\frac{w \cosh (w t)+c \lambda \sinh (w t)}{w} & \frac{\left(A_{2}+\lambda A_{1}\right) \sinh (w t)}{2 w} \\
\frac{-\left(A_{2}-\lambda A_{1}\right) \sinh (w t)}{2 w} & \frac{w \cosh (w t)-c \lambda \sinh (w t)}{w}
\end{array} \mid\right.
$$

When $w^{2}<0$

$$
E_{i j}=\left(\begin{array}{cc}
\frac{w \cos (w t)+c \lambda \sin (w t)}{w} & \frac{\left(\lambda A_{1}+A_{2}\right) \sin (w t)}{2 w} \\
\frac{\left(\lambda A_{1}-A_{2} \sin (w t)\right.}{2 w} & \frac{w \cos (w t)-c \lambda \sin (w t)}{w}
\end{array} \mid\right.
$$

These three categories of solutions represent the effect of flow kinematics on fiber orientation and using them in Equation (6) would give the orientation vector component. Substituting $\lambda=1$ simplifies these equations for the infinite aspect ratio fibers and the three different $E_{i j}$ 's represent the strain tensors for simple shear, planar elongation, and rotation dominant flows respectively.

Using the strain tensor equations above with Equation (6), a number of fibers, each starting from different orientation, are traced along the particle paths for all the grid points. At the inlet, random fiber orientation is implemented by considering a number of discrete fibers oriented with equal angular intervals between 0 and $\pi$. In this study, nine and ninety fibers are utilized in the solution. Therefore, at the inlet, the angular intervals between nine and ninety fibers become $\pi / 9$ and $\pi / 90$ respectively. After being subjected to a local velocity gradient, a new set of fiber orientations are calculated describing a statistical orientation distribution. Obviously, as the number of fibers is increased, more accurate representation of the orientation distribution will be obtained. From such an orientation distribution, the preferred angle is taken to be the midpoint of the smallest angular interval within a particular set of fiber orientations.

\section{RESULTS}

For both converging and diverging channels, orientation results are obtained from four different methods, using:

(1) Second-order orientation tensors

(2) Fourth-order orientation tensors

(3) Statistical orientation distribution by tracing nine fibers

(4) Statistical orientation distribution by tracing ninety fibers

For these four methods, two different fiber aspect ratios are considered:

(1) $a_{p} \rightarrow \infty$

(2) $a_{p}=10$ 


\section{Converging Channel Geometry}

A forty-five-degree convergent channel as shown in Figure 1 is chosen for the analysis. All lengths are non-dimensionalized with respect to the straight channel width, and the total channel length is selected to be ten. Due to the high extensional velocity gradients, the grid lines are concentrated towards the contraction of the channel as shown in Figure 2. Figure 3 provides a comparison of the velocity profiles at the contraction of the channel for the various grid sizes. After numerous runs with different grid sizes and convergence criteria, a mesh size of $37 \times 21$ and a relative convergence criteria of $10^{-6}$ are found to yield accurate results.

The orientation results are presented as graphs showing the variation of the maximum orientation angles across the channel width at different sections of the channel as in Figures $4 a-4 c$ and $6 a-6 c$, and the variation of orientation angles along the channel length as in Figures $4 \mathrm{~d}$ and $6 \mathrm{~d}$. In addition, Figures $5 \mathrm{a}-5 \mathrm{c}$ and $7 \mathrm{a}-7 \mathrm{c}$, show the preferred fiber orientations throughout the domain of interest. Tracing ninety fibers in the statistical solution is found to adequately represent the orientation field while considering only nine fibers gave some discrepancies. A further increase in the number of fibers did not yield results drastically different from those obtained for ninety fibers. Maximum orientation angle, $\phi_{\max }$, is calculated as explained before, and comparison of the results obtained from the second- and fourth-order orientation tensor solutions [i.e., from Equations (37) and (38)] revealed identical $\phi_{\max }$ values for all the grid points. Hence, one can conclude that both evolution equations for the second- and fourth-order orientation tensor yields correct orientation angles despite the quadratic closure and approximation. Of course this conclusion can be drawn considering the fact

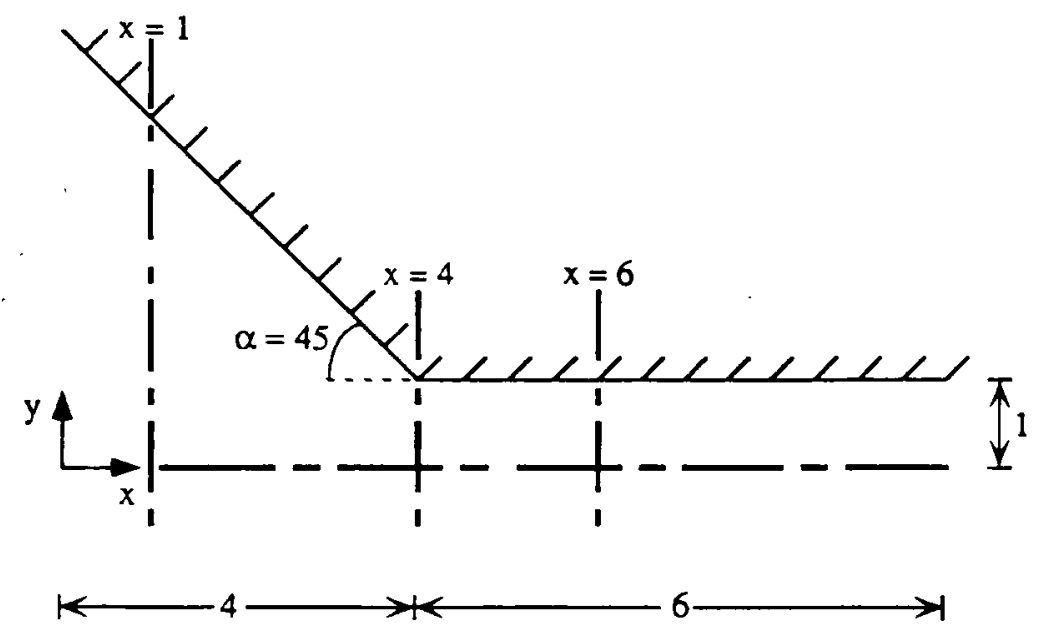

Figure 1. Converging channel geometry. 


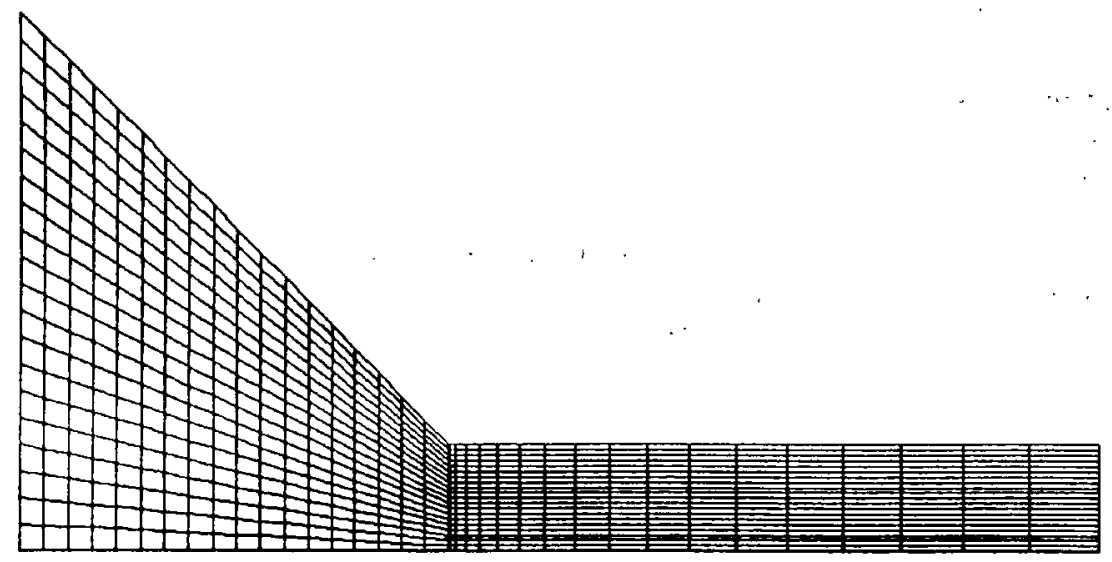

Figure 2. Mesh chosen for the converging channel. Mesh size: $37 \times 21$.

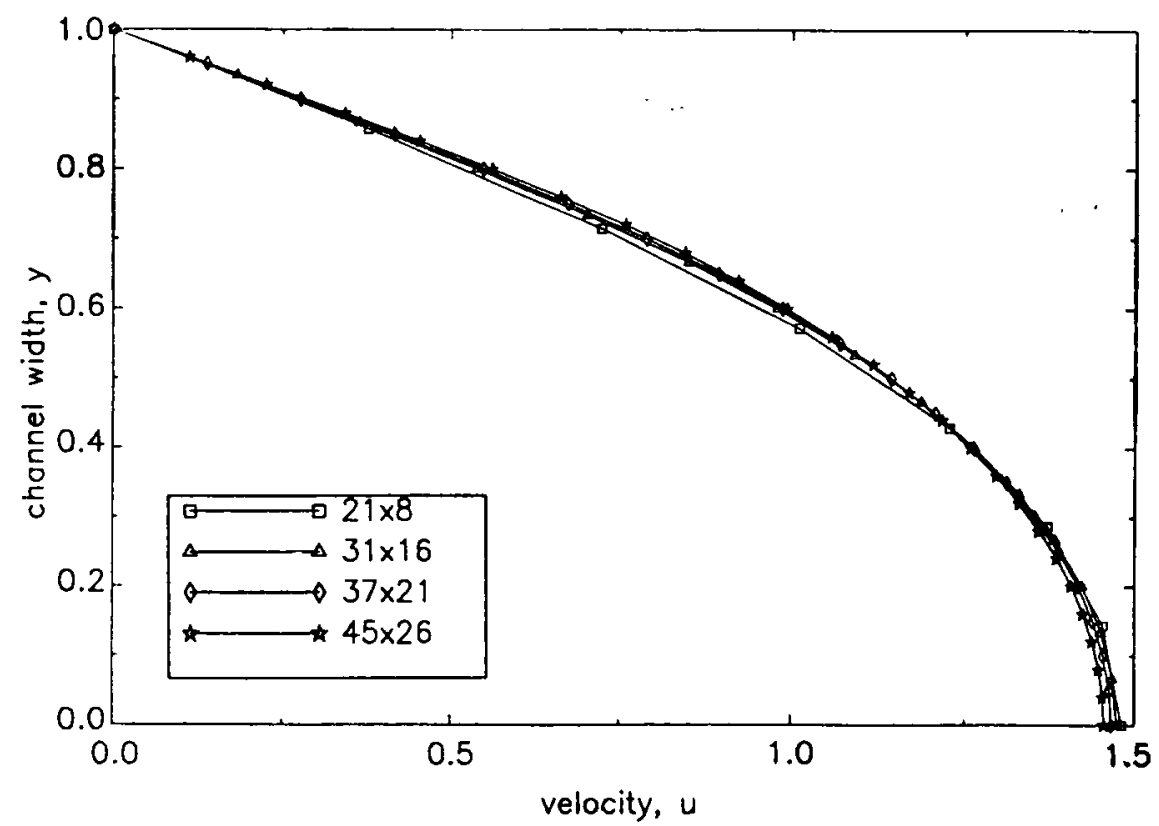

Figure 3. Variation of the $u$ component of the velocity across the channel width for different mesh sizes. 


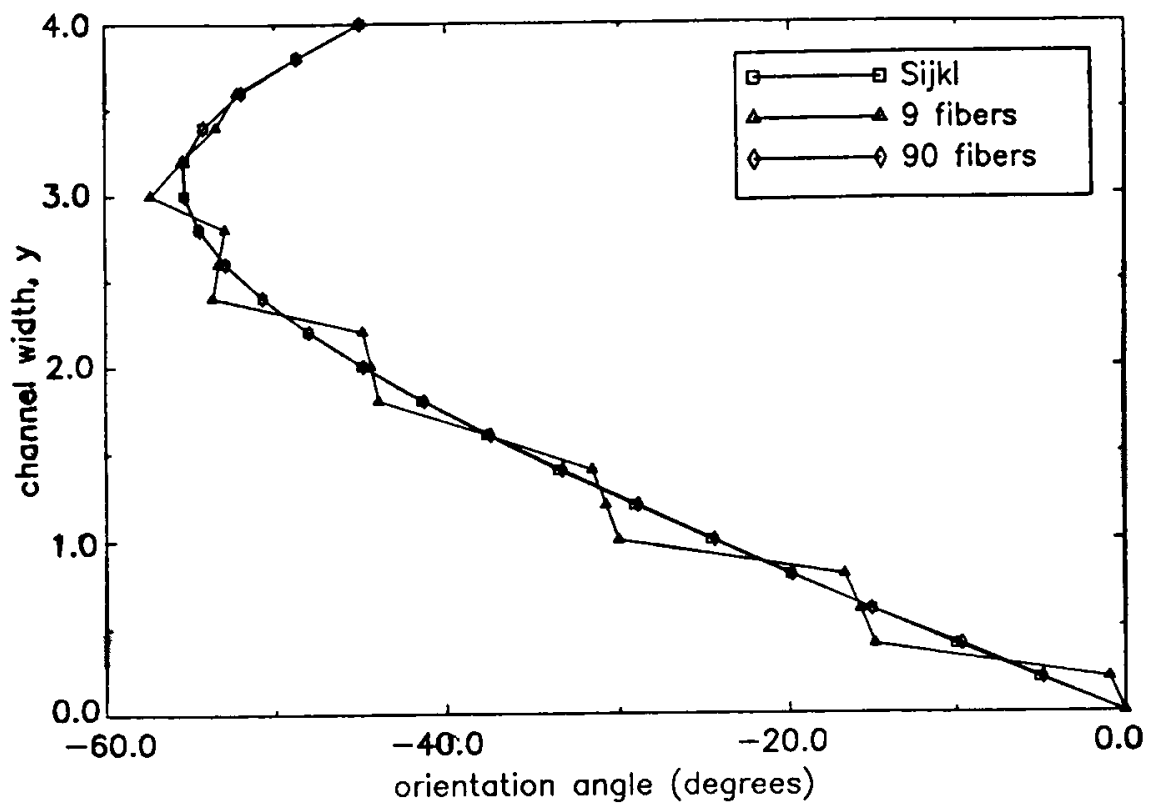

Figure 4a. Variation of orientation angle across the channel width close to the inlet of the converging channel at $x=1$, for infinite aspect ratio fibers.

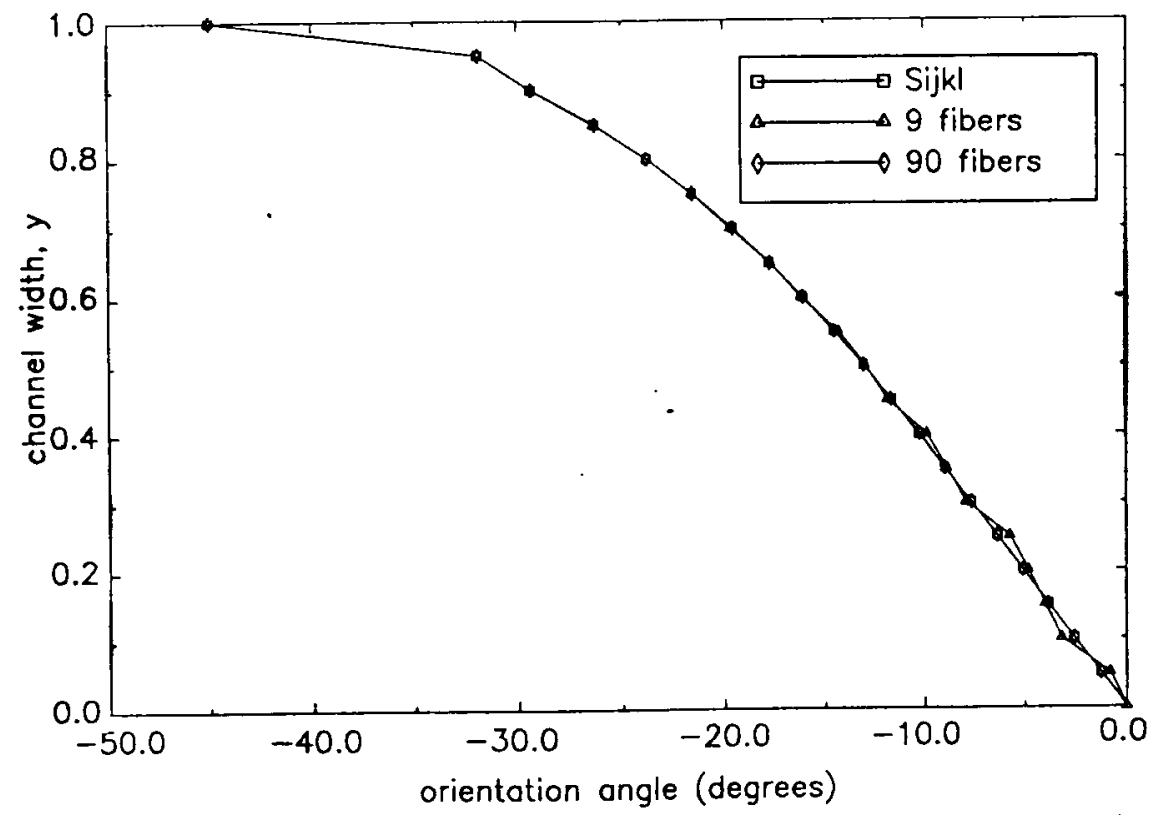

Figure 4b. Variation of the orientation angle across the channel width at the contraction section of the converging channel at $x=4$, for infinite aspect ratio fibers. 


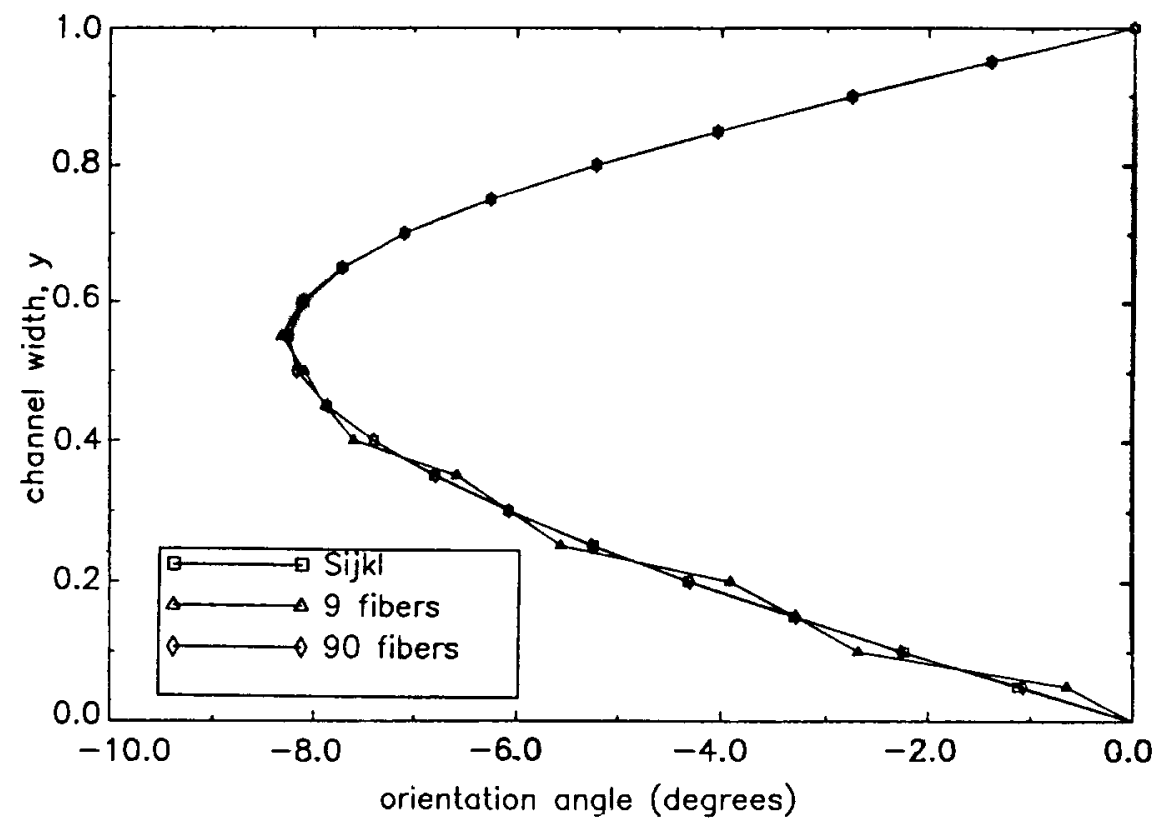

Figure 4c. Variation of orientation angle across the channe/ width at some distance from the contraction section of the converging channel at $x=6$, for infinite aspect ratio fibers.

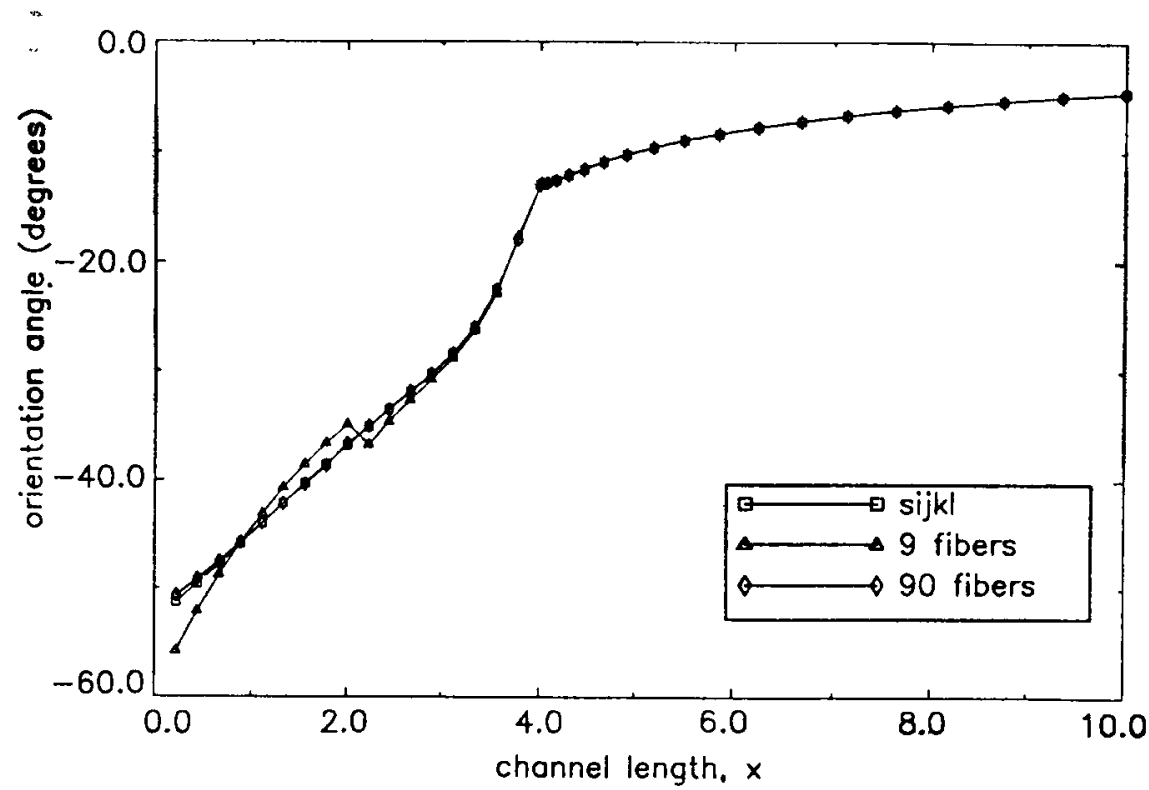

Figure 4d. Variation of orientation angle along the channel length at the midpoint of the channel width with infinite aspect ratio fibers. 


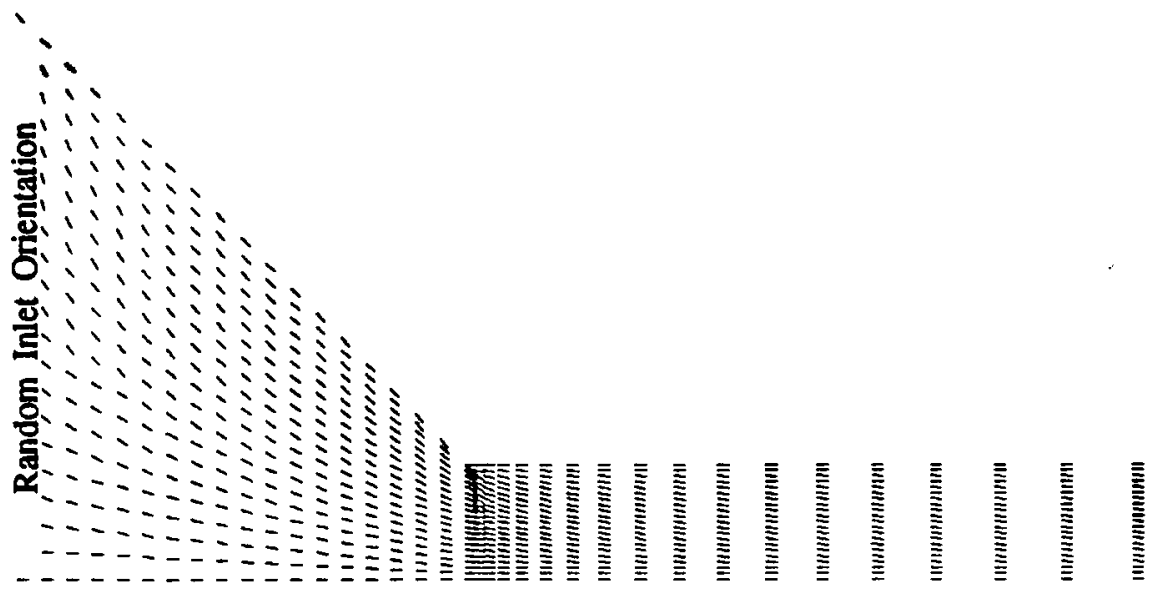

Figure 5a. Orientation angles of infinite aspect ratio fibers in the converging geometry obtained by tracing nine fibers.

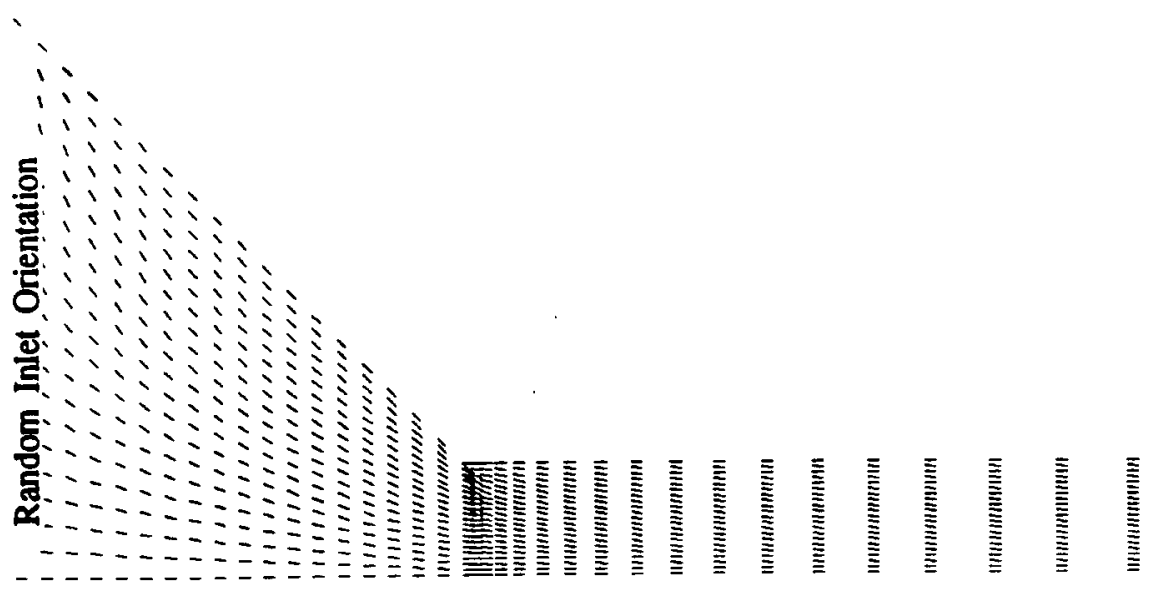

Figure $5 \mathrm{~b}$. Orientation angles of infinite aspect ratio fibers in the converging geometry obtained by tracing ninety fibers. 
1112

동!i i i

Gi i i i :

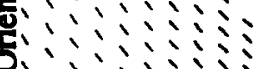

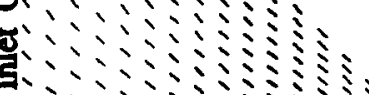

E⿺辶ン:

Figure 5c. Orientation angles of infinite aspect ratio fibers obtained from the solution of fourth-order orientation tensor $S_{i k j}$.

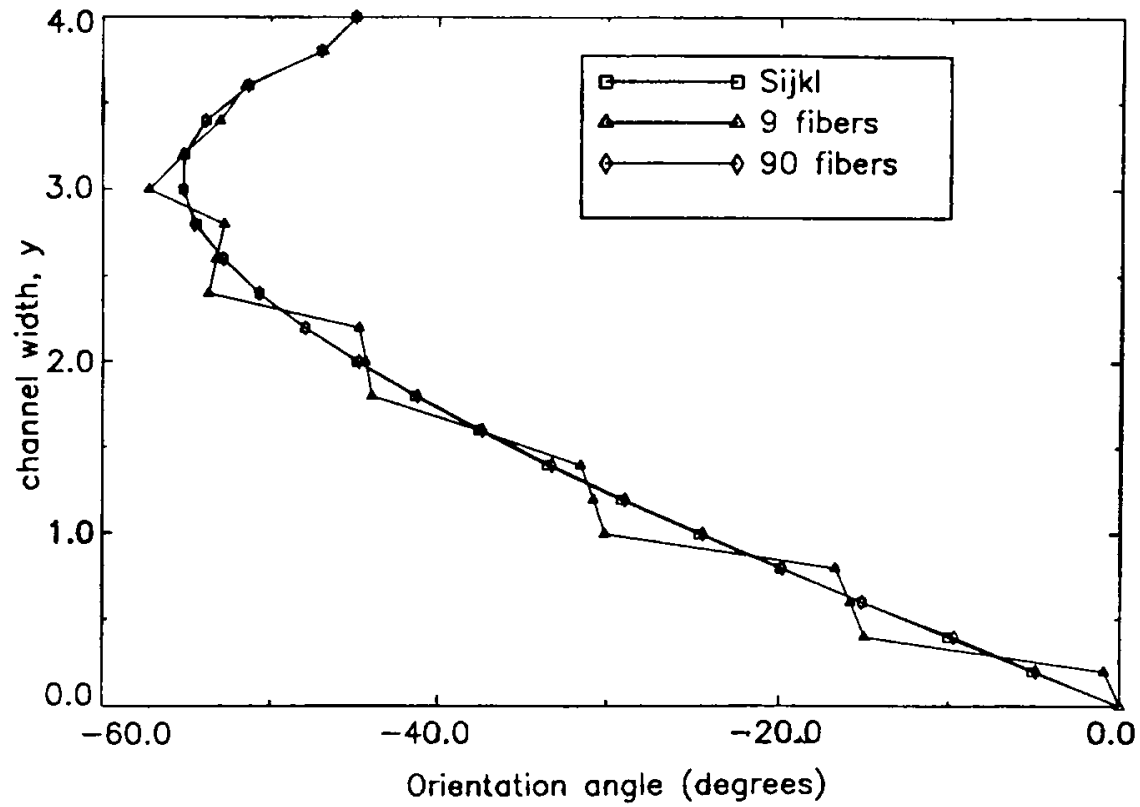

Figure 6a. Variation of orientation angle across the channel width close to the inlet of the converging channel at $x=1$, for $a_{\rho}=10$. 


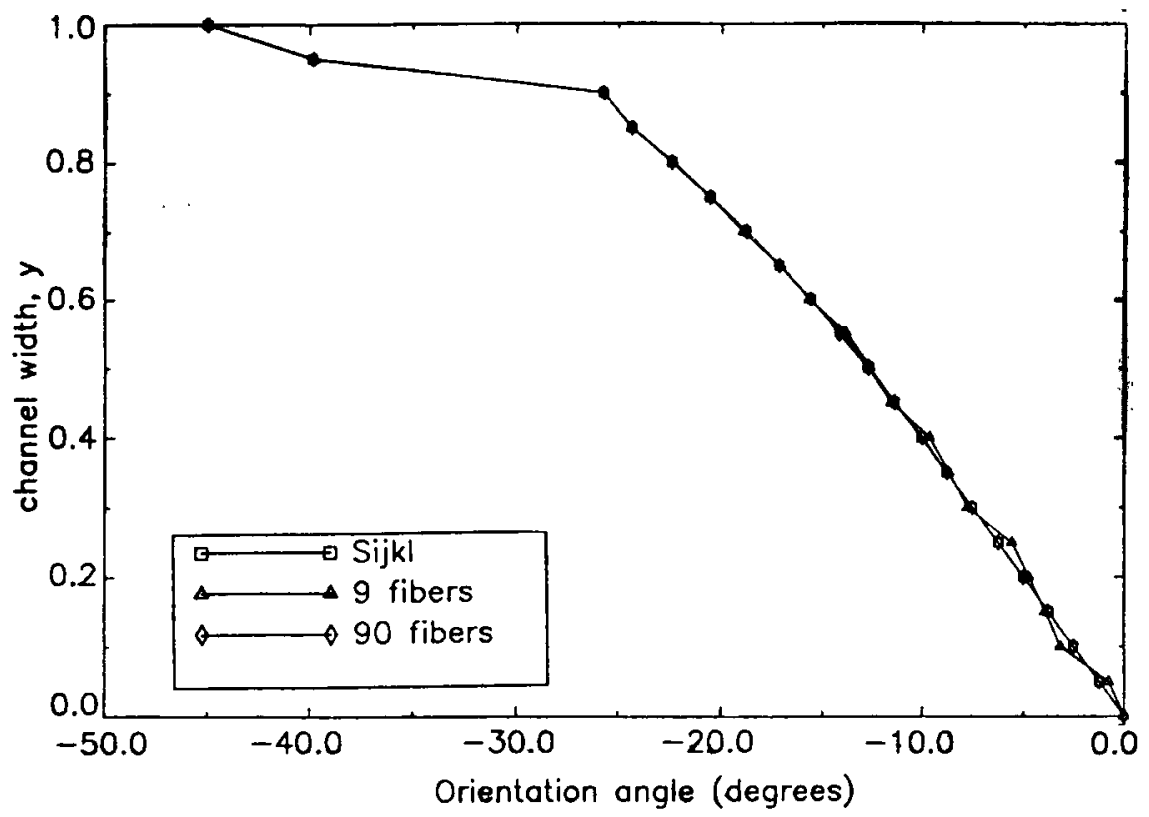

Figure 6b. Variation of orientation angle across the channe/ width at the contraction section of the converging channel at $x=4$, for $a_{p}=10$.

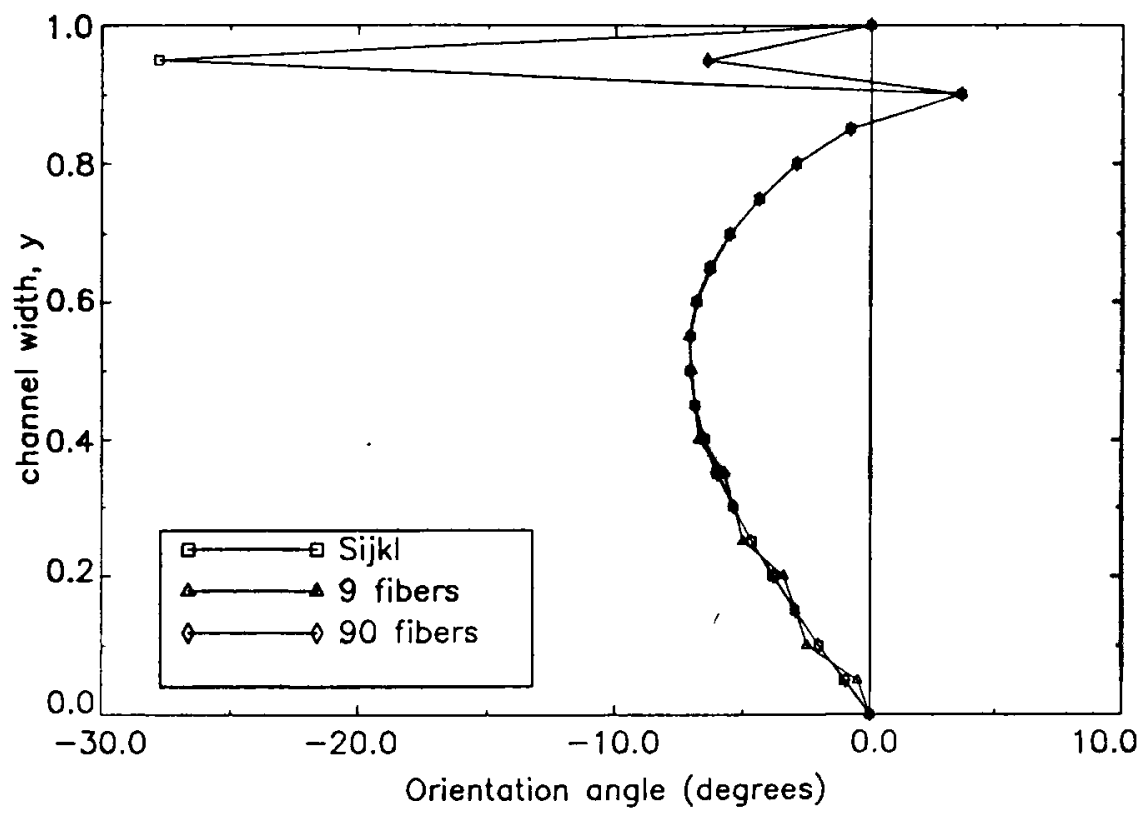

Figure 6c. Variation of orientation angle across the channel width at some distance from the contraction section of the converging channel at $x=6$, for $a_{p}=10$. 


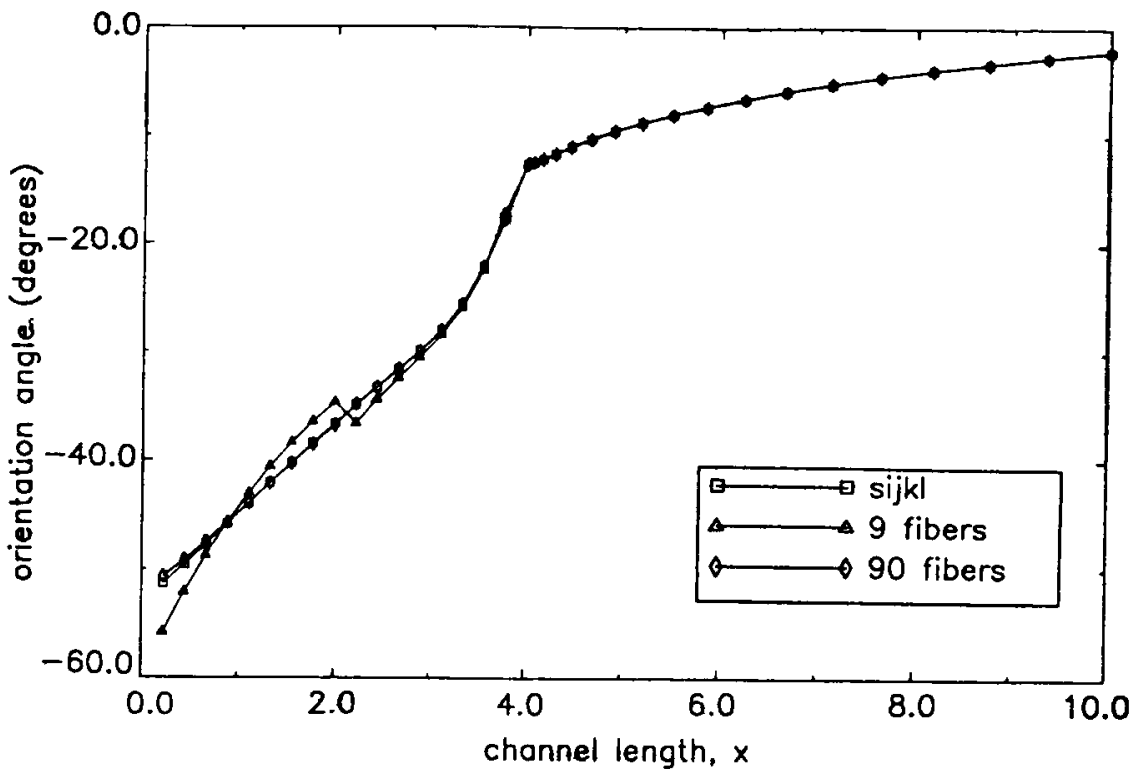

Figure $6 d$. Variation of orientation angle along the channel length at the midpoint of the channel width for $a_{p}=10$.

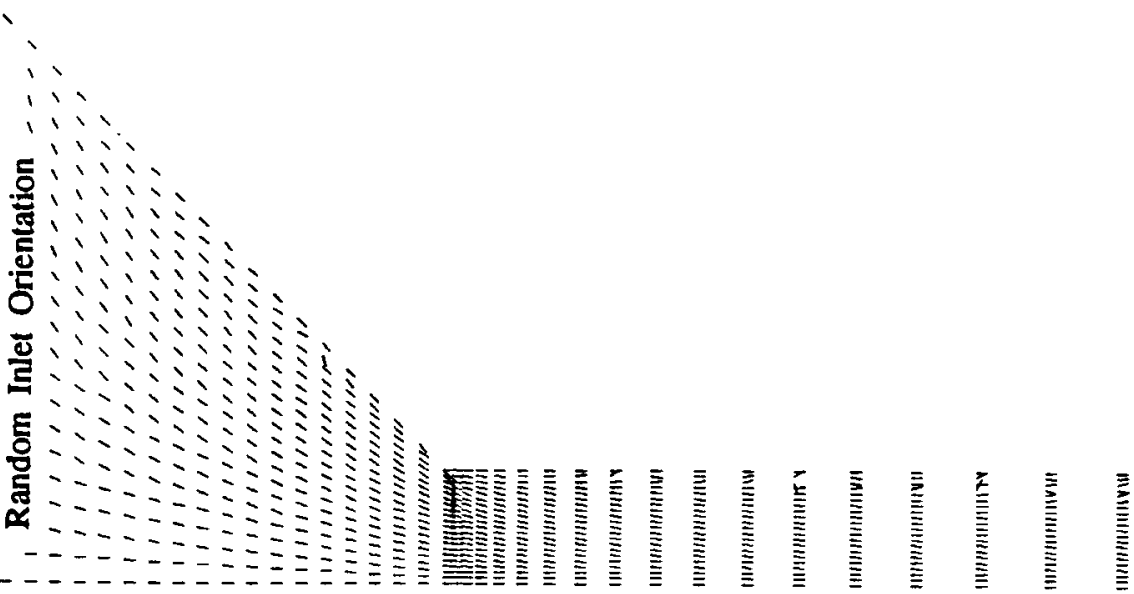

Figure 7a. Orientation angles of finite aspect ratio fibers $\left(a_{p}=10\right)$ in the converging geometry obtained by tracing nine fibers. 


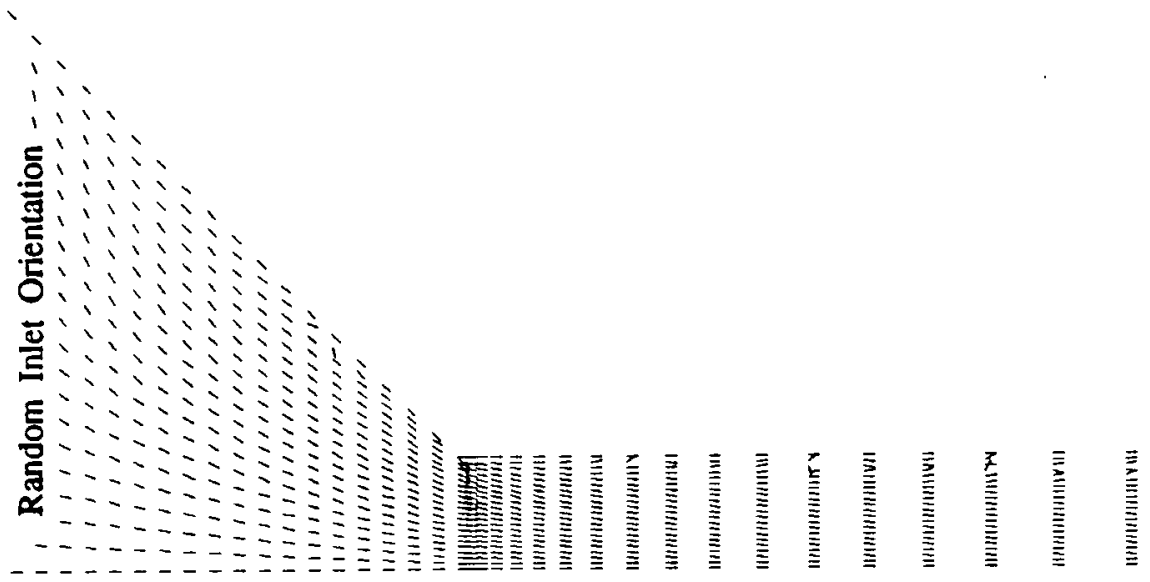

Figure $7 b$. Orientation angles of finite aspect ratio fibers $\left(a_{p}=10\right)$ in the converging geometry obtained by tracing ninety fibers.

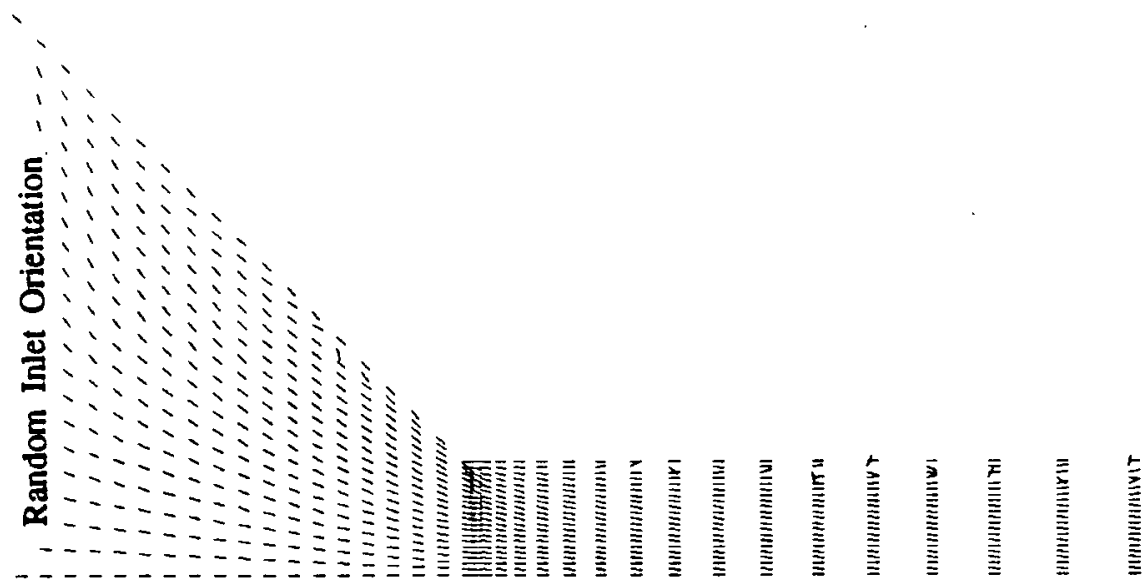

Figure 7c. Orientation angles of finite aspect ratio fibers $\left(a_{p}=10\right)$ obtained from the solution of fourth-order orientation tensor $S_{\eta \mid k l}$. 
that for an $n$ th-order tensor, the results should approach to exact values as $n$ approaches infinity. Consequently, in this paper, only the results from the fourthorder orientation tensors were used in the graphs and vector plots.

Figures 4a and 6a show the maximim orientation angles obtained close to the inlet of the converging channel. Figure $4 a$ depicts the results for infinite fiber aspect ratio, while Figure 6a depicts the results for an aspect ratio of ten. Except for a small difference in the orientation curves observed near the channel wall, the orientation results in these two figures [Figures $4 a$ and $6 a$ ] are seen to be almost identical. Since the elongational component of the flow is dominant in the converging section of the channel, the fibers try to align rapidly along the radial lines and no tumbling is observed. Clearly, the solution obtained by tracing ninety fibers is seen to approach to the fourth-order tensor solution whereas the solution with nine fibers shows a zigzag pattern over the other two curves. For all practical purposes, utilizing more than ninety fibers would only cause the statistical solution to give preferred orientations identical with those obtained from the orientation tensors. Figures $4 \mathrm{~b}$ and $4 \mathrm{c}$ depict the preferred orientation for infinite aspect ratio fibers at the contraction and at some distance away from the contraction. Correspondingly, Figures $6 \mathrm{~b}$ and $6 \mathrm{c}$ represent the preferred orientations for the fibers with aspect ratio of ten. At the contraction, all the fibers enter the straight channel more or less coincident with the radial lines (Figures 5 and 7). However, from the comparison of Figures $4 b$ and $6 \mathrm{~b}$, the orientation angles are observed to be slightly different near the channel wall. In the straight channel portion, the shear flow is prominent, and as expected, the fibers tend to align themselves with the flow as seen in Figure 4c. Due to high shear rates existing near the wall of the channel, the fibers close to the wall align themselves at a quicker rate (Figure 5) compared to those close to the centerline of the channel. On the other hand, the finite aspect ratio solution depicts fiber tumbling near the channel wall, as seen in Figures $6 \mathrm{c}$ and 7. As shown in Figure 6c, the agreement between the statistical and tensorial solutions is satisfactory except for one node near the wall. A better illustration of the variation in orientation angles along the channel length can be seen in Figures $4 \mathrm{~d}$ and $6 \mathrm{~d}$. Note that the orientation angles in Figure $4 \mathrm{~d}$ and $6 \mathrm{~d}$ are obtained at the midpoints of the half channel width throughout the domain. A close look at these two figures reveals that the finite aspect ratio fibers align more rapidly compared to more stable infinite aspect ratio fibers. Although not much difference in the orientation angles is visible in the straight channel part of the converging geometry, a careful examination of Figures $5 \mathrm{a}, 5 \mathrm{~b}$ and $5 \mathrm{c}$ in the converging portion of the channel shows some difference in the orientation angles between the solutions obtained by tracing nine fibers and the other two solutions. In contrast, Figures $7 \mathrm{a}, 7 \mathrm{~b}$ and $7 \mathrm{c}$ depict the orientation tensor results to be somewhat different from the other two results in a region close to the wall. These discrepancies observed in Figures 7 and $6 \mathrm{c}$ are mainly associated with the fibers that are rotating rapidly within a smaller length scale than the mesh size. In other words, the fibers are subjected to relatively high shear rates near the channel wall that result in rapid tumbling over much smaller the negative to positive orientation could be attributed to the expanding and rapidly decelerating flow at the inlet of the diverging section, while the second cross- 
length scales. Therefore, with the fixed nonadaptive mesh, orientation predictions may not be very accurate for such regions of high orientation gradients.

\section{Diverging Channel Geometry}

A diverging channel geometry as shown in Figure 8 is selected for the analysis. All lengths are non-dimensionalized with respect to the width of the channel at the inlet and the overall channel length is chosen to be twelve. A straight channel is included after the diverging section for ease in satisfying the exit boundary conditions. A mesh size of $45 \times 31$ with a relative convergence criteria of $10^{-6}$ is used in the computations. Due to the high velocity gradients near the inlet and exit of the diverging section, grid concentration in these regions is found to be necessary. In addition, the grid lines are concentrated near the channel wall as shown in Figure 9 in an attempt to capture the possible fiber tumbling in detail. Figures $10 \mathrm{a}$ and $12 \mathrm{a}$ show the variation of the orientation angle across the channel width at the inlet of the diverging section for infinite and finite aspect ratio (i.e., $a_{p}=10$ ) fibers respectively. At this section, due to the effect of the shear rates throughout the straight channel, the fibers near the channel wall started to align along the flow direction. However, due to the zero shear and negative extensional velocity gradients on the centerline, the preferred orientation angles are seen to be perpendicular to the flow. Obviously, this is due to the diverging section through which the fluid decelerates. The preferred orientations obtained by tracing nine fibers are observed to be sufficiently accurate as shown in Figures $10 \mathrm{a}-10 \mathrm{e}$. Increasing the number of fibers from nine to ninety only improved the results slightly. The effect of the diverging geometry is much more prominent in

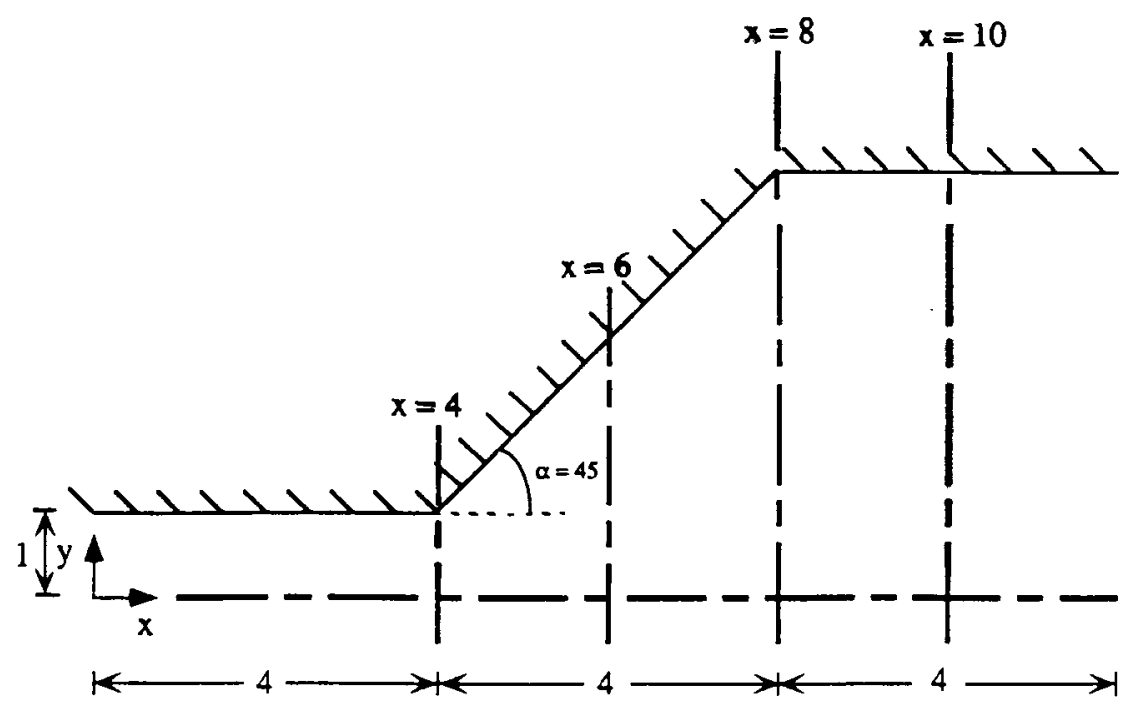

Figure 8. Diverging channel geometry. 


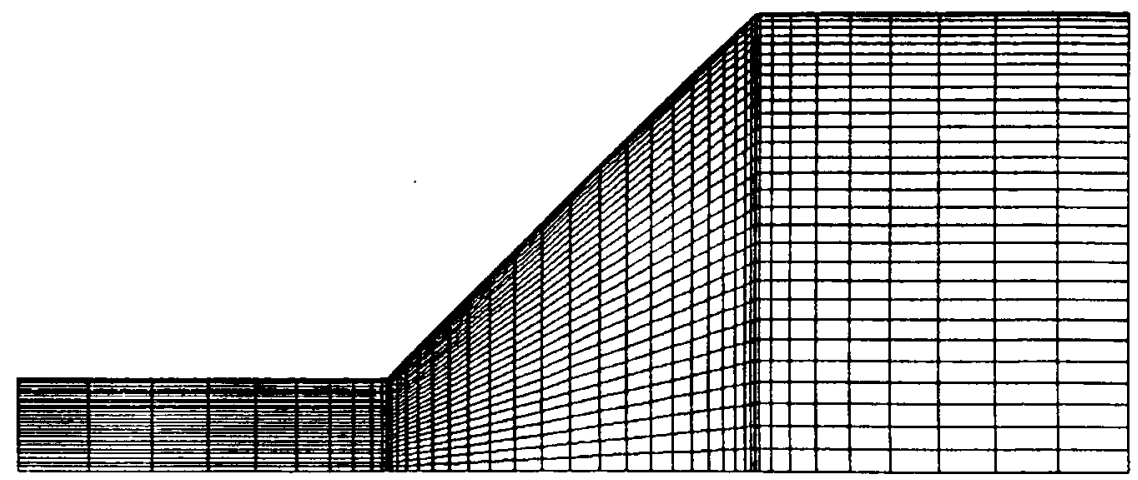

Figure 9. Mesh chosen for the diverging channel. Mesh size: $45 \times 31$.

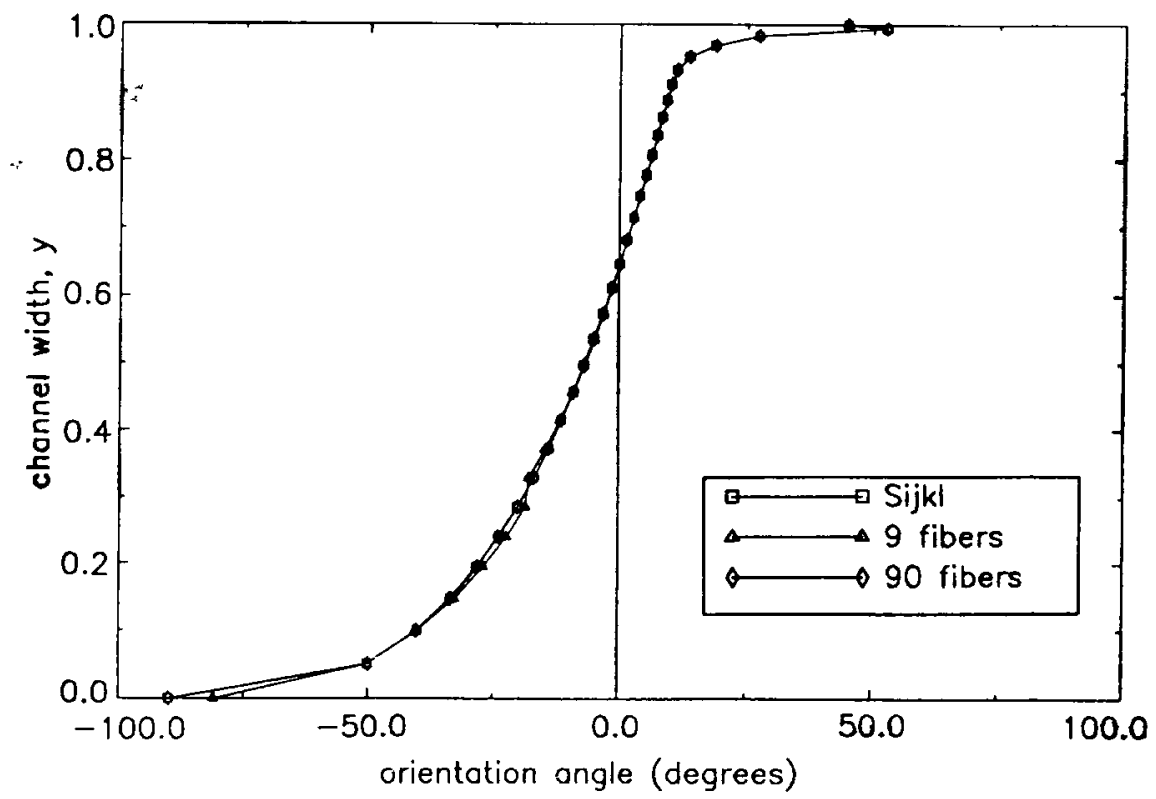

Figure 10a. Variation of orientation angle across the channel width at the beginning of the diverging section at $x=4$, for infinite aspect ratio fibers. 


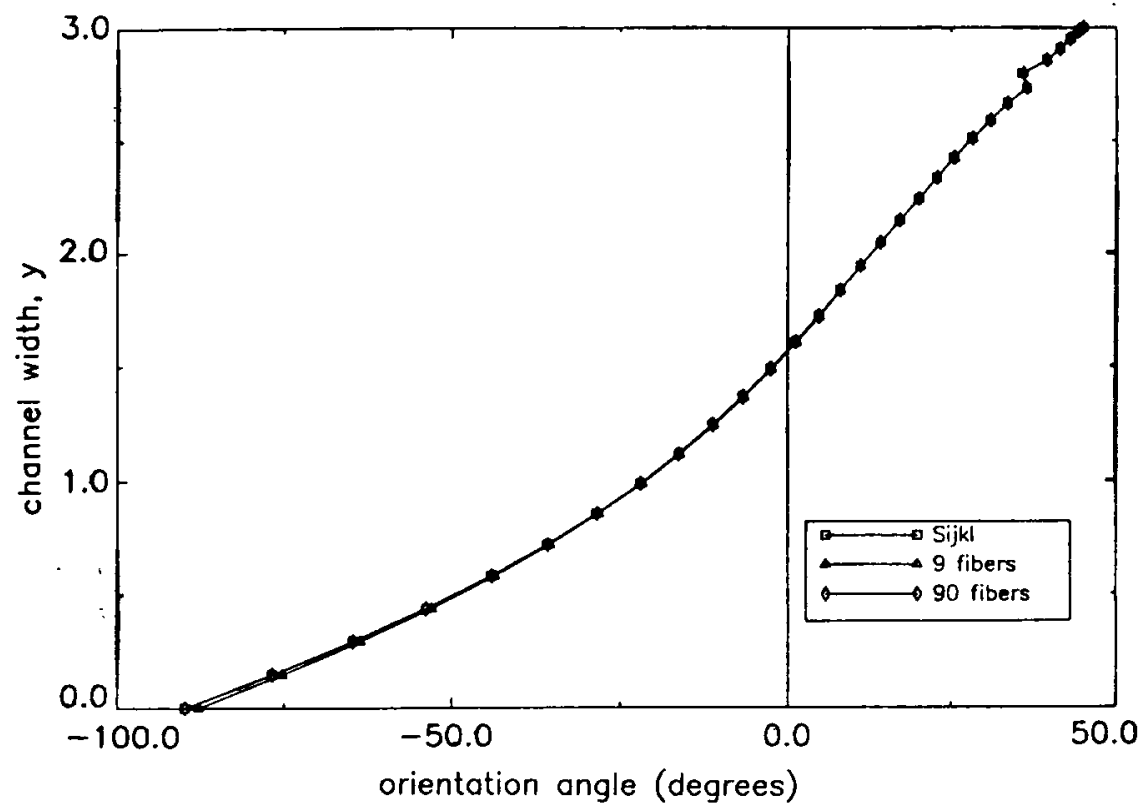

Figure 10b. Variation of the orientation angle across the channel width midway through the diverging section at $x=6$, for infinite aspect ratio fibers.

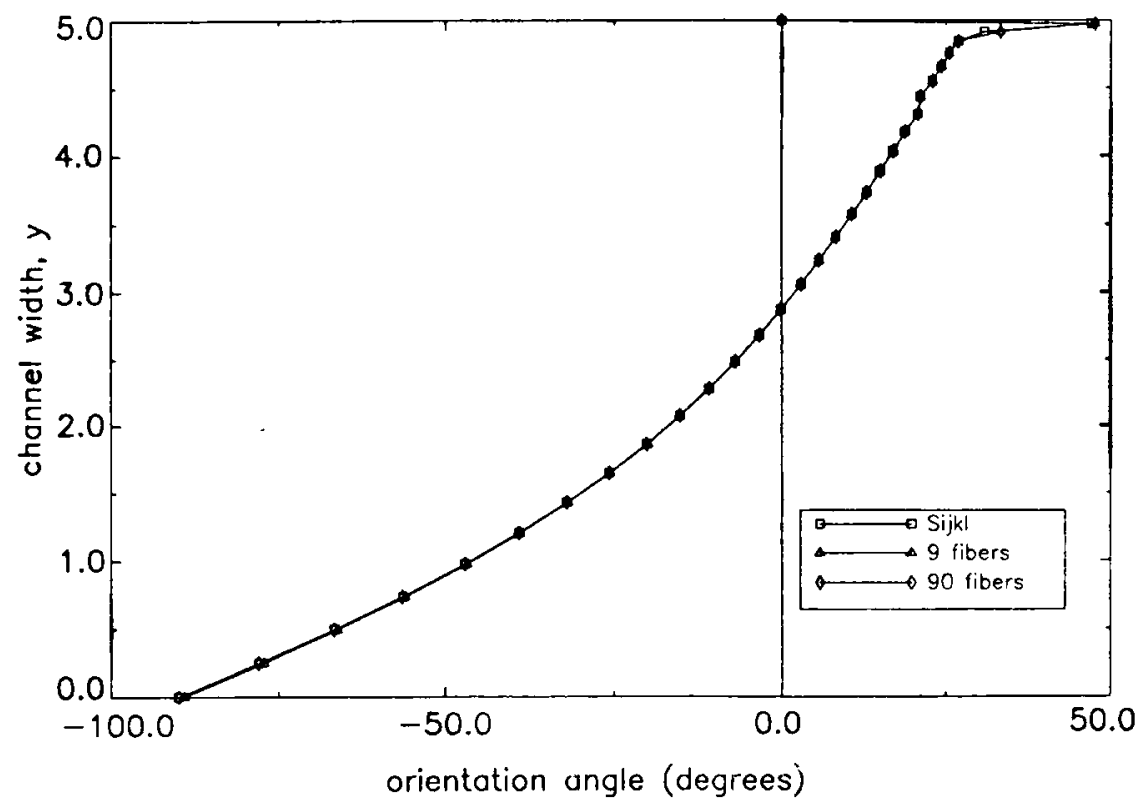

Figure 10c. Variation of orientation angle across the channel width at the end of the diverging section at $x=8$, for infinite aspect ratio fibers. 


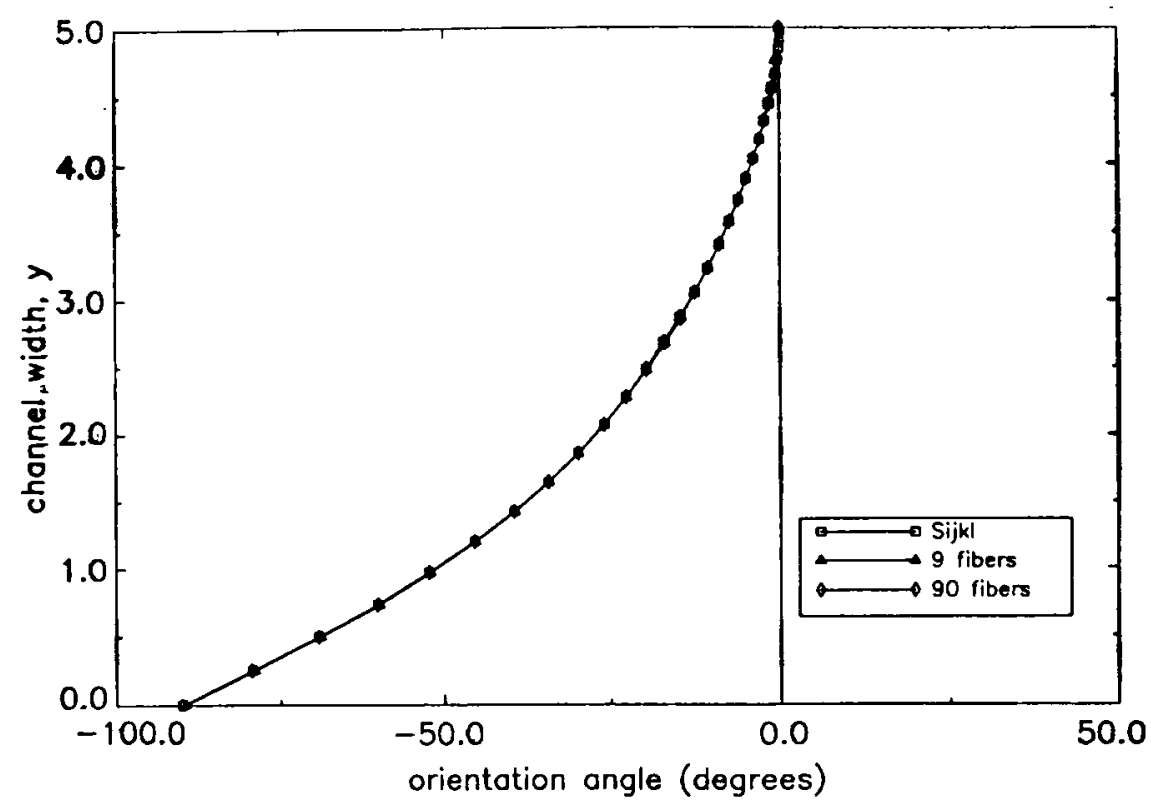

Figure 10d. Variation of orientation angle across the channel width at some distance from the end of the diverging section at $x=10$, for infinite aspect ratio fibers.

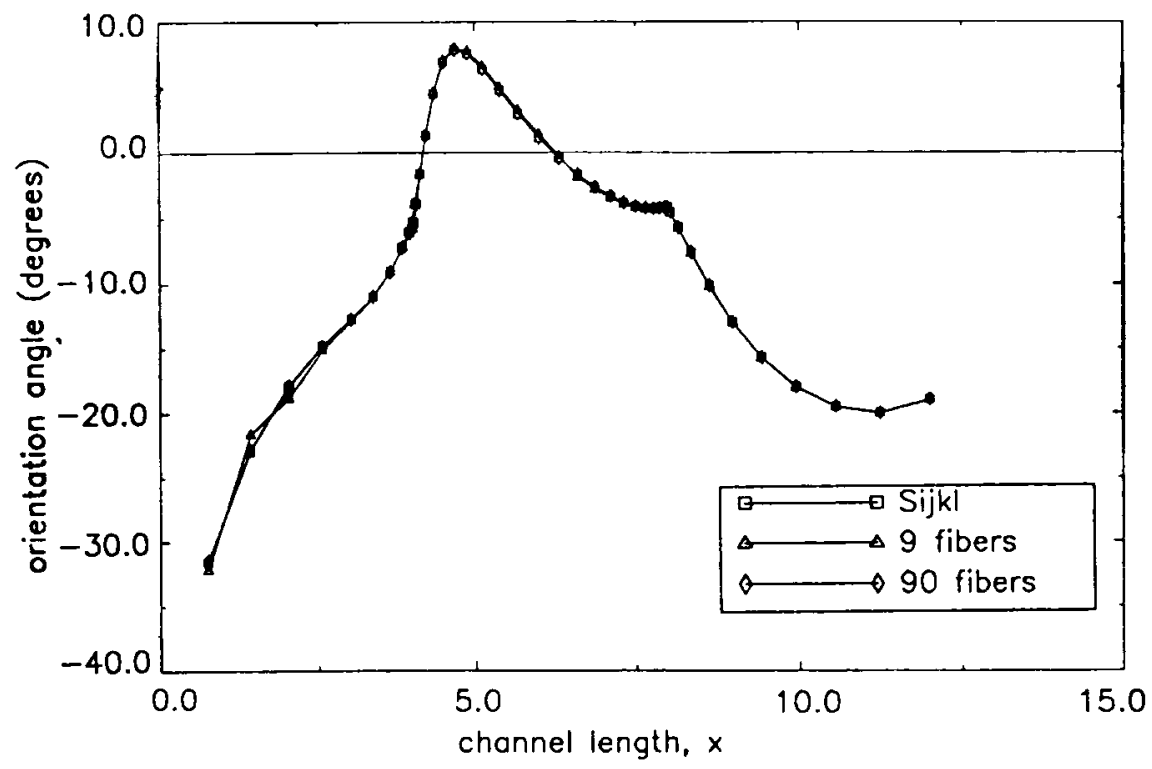

Figure 10e. Variation of orientation angle along the channel length at the midpoint of the channel width, for the diverging channel with infinite aspect ratio fibers. 


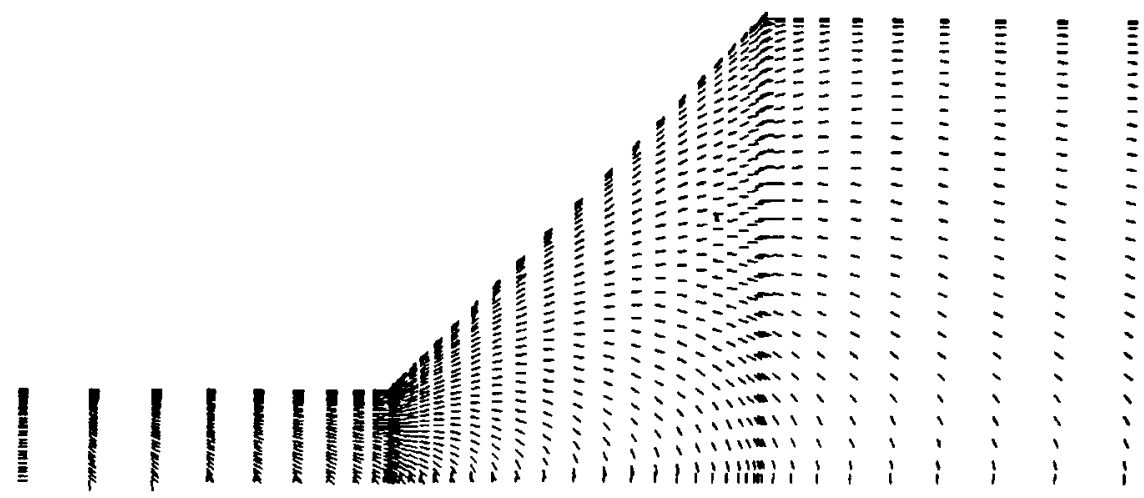

Figure 11a. Orientation angles of infinite aspect ratio fibers in the diverging geometry obtained by tracing nine fibers.

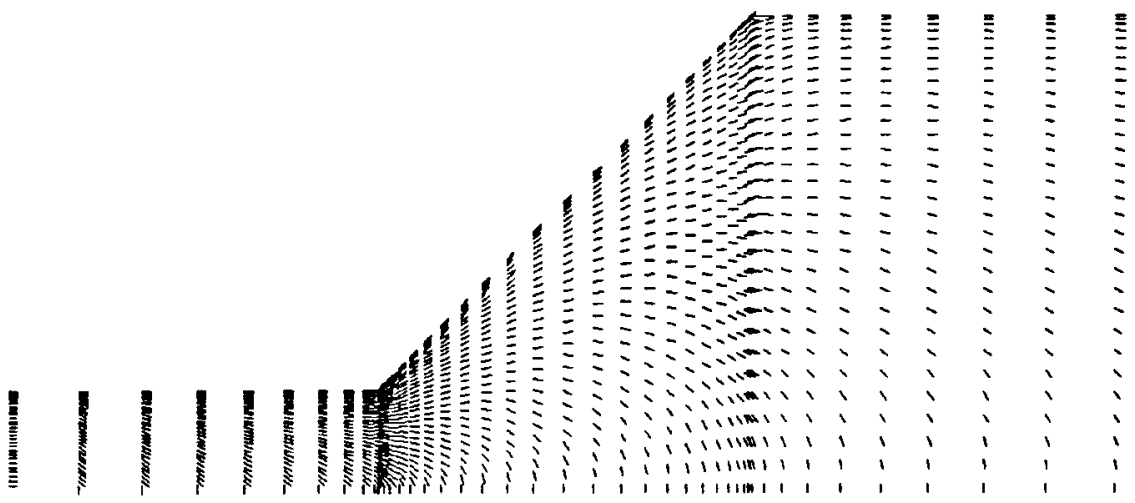

Figure 11b. Orientation angles of infinite aspect ratio fibers in the diverging geometry obtained by tracing ninety fibers. 


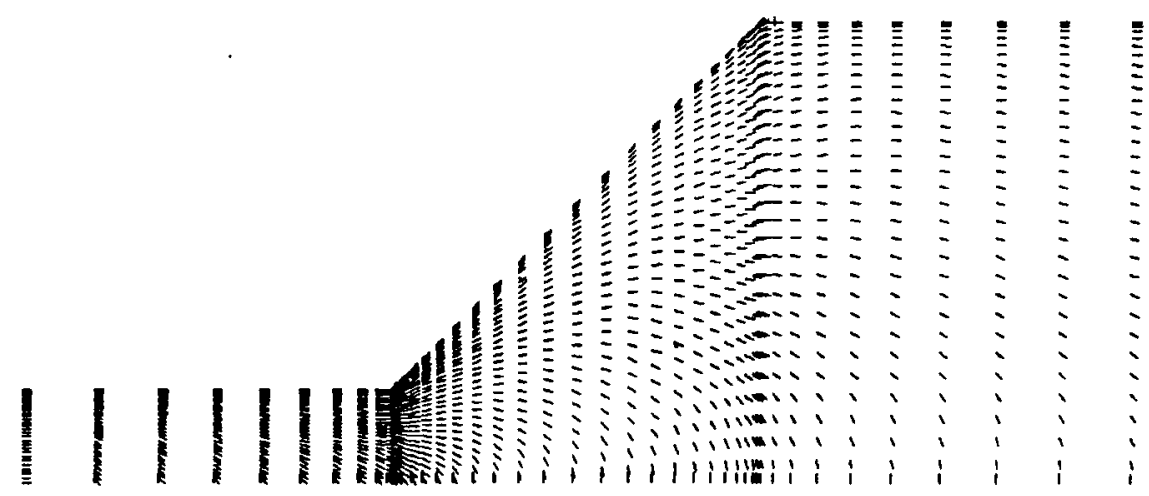

Figure 11c. Orientation angles of infinite aspect ratio fibers obtained from the solution of fourth-order orientation tensor $S_{i k k}$.

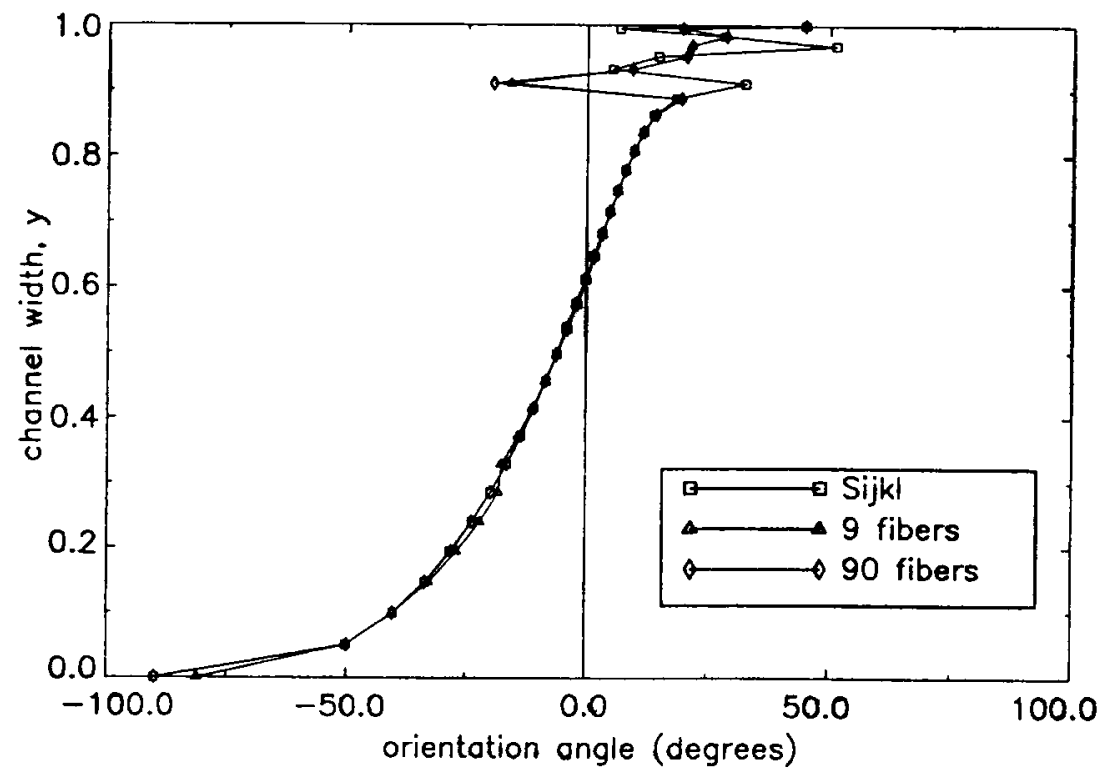

Figure 12a. Variation of orientation angle across the channel width at the beginning of the diverging section at $x=4$, for $a_{p}=10$. 


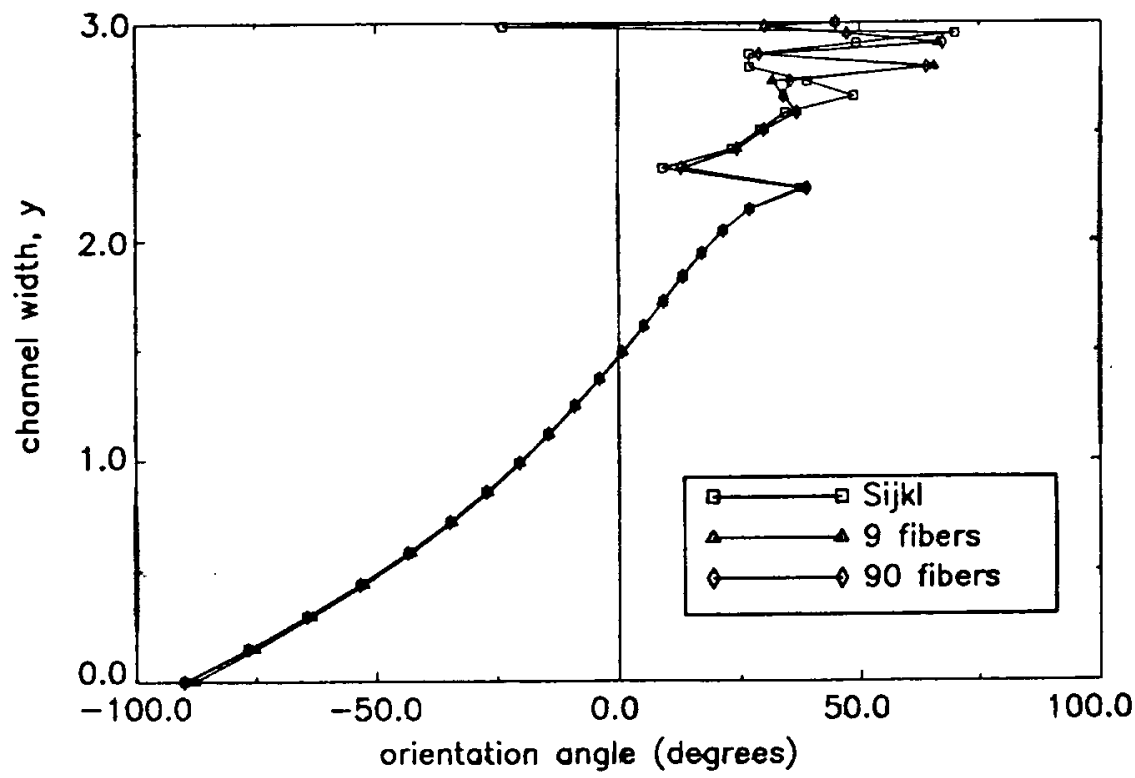

Figure 12b. Variation of orientation angle across the channel width midway through the diverging section at $x=6$, for $a_{p}=10$.

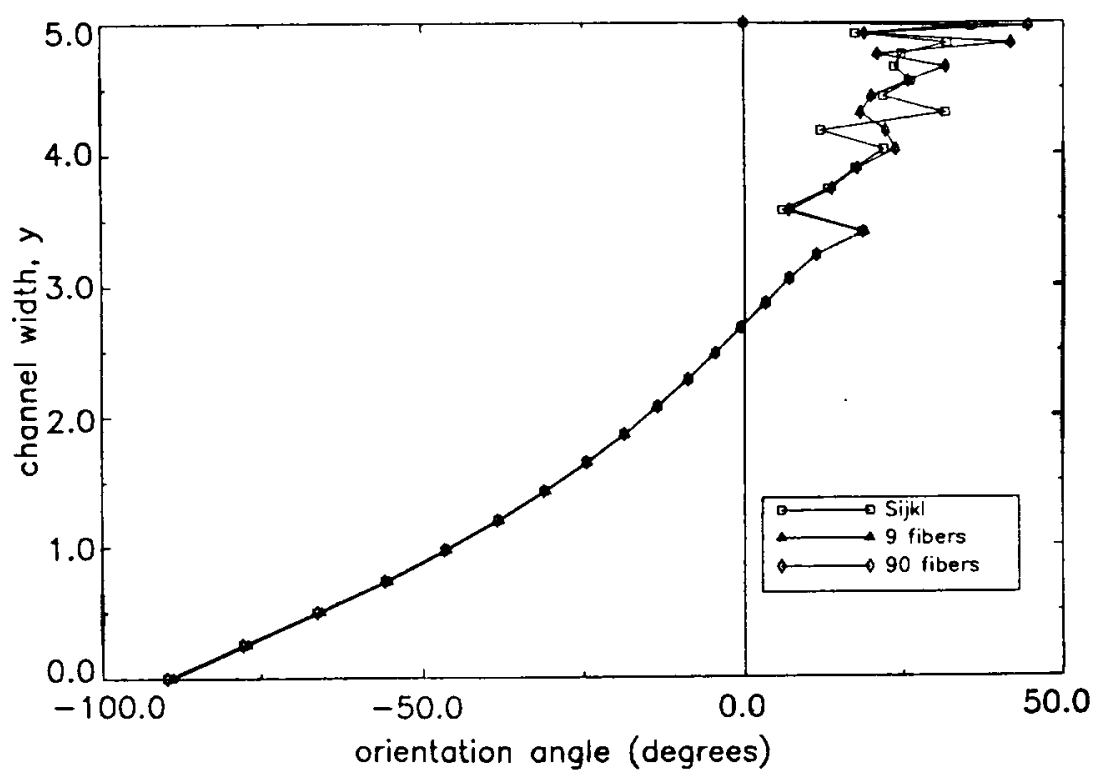

Figure 12c. Variation of orientation angle across the channel width at the end of the diverging section at $x=8$, for $a_{p}=10$. 


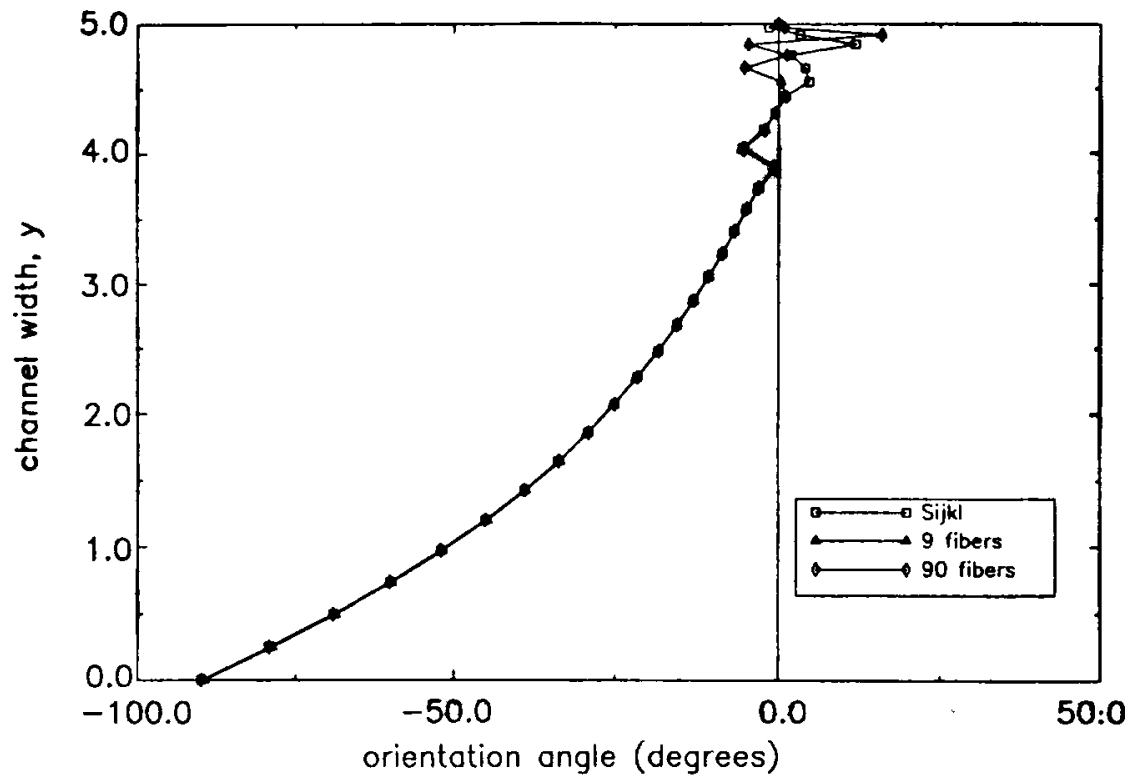

Figure 12d. Variation of orientation angle across the channel width at some distance from the end of the diverging section at $x=10$, for $a_{p}=10$.

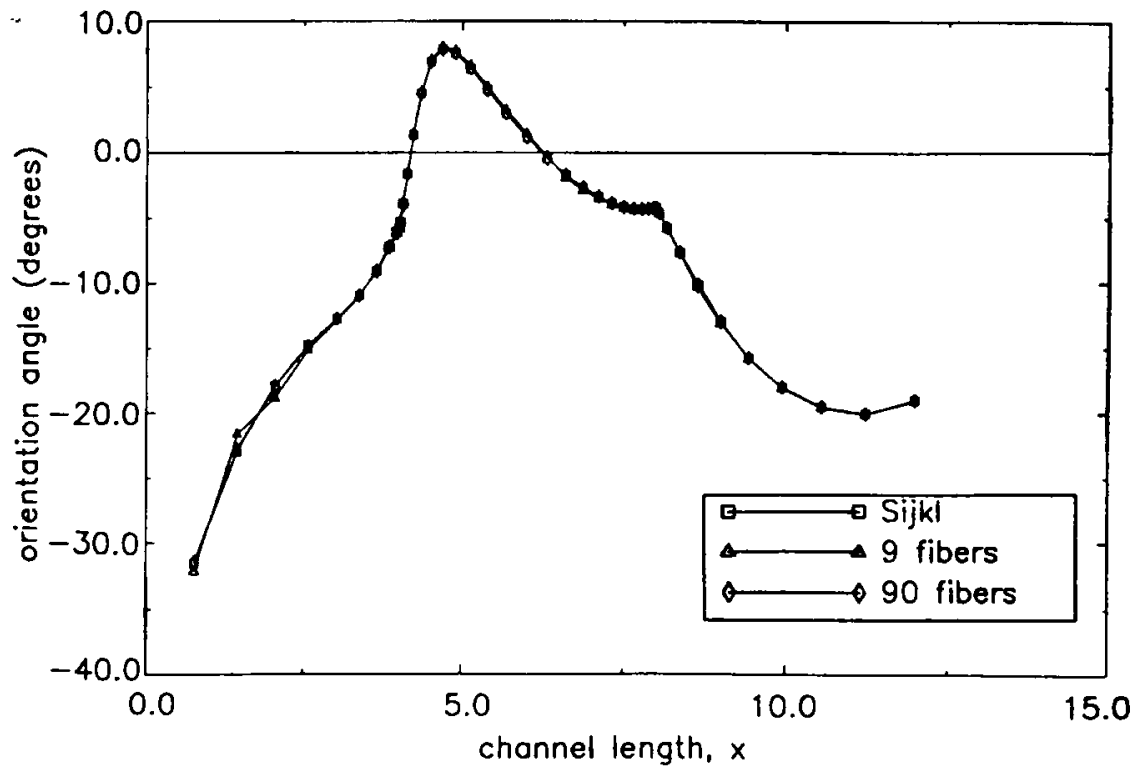

Figure 12e. Variation of orientation angle along the channel length at the midpoint of the channel width of the diverging channel for $a_{p}=10$. 
Figures $10 \mathrm{~b}$ and 10c [see also Figures 1la-11c] which are obtained for infinite fiber aspect ratios, and in Figures $12 \mathrm{~b}$ and $12 \mathrm{c}$ [see also Figures 13a-13c], which are obtained for fibers with aspect ratio of ten. At these sections, which are the center and the exit of the diverging portion of the channel respectively, most fibers close to the centerline orient themselves almost perpendicular to the flow direction. Results for infinite aspect ratio fibers, as shown in Figures 10b-10c, indicate a rather smooth transition from a preferred orientation of $-90^{\circ}$ at the centerline to $45^{\circ}$ at the wall. The results for fibers with an aspect ratio of ten, as shown in Figures 12b-12c, indicate a similar transition; however, due to high shear gradients throughout a narrow band near the channel wall, the finite aspect ratio fibers are seen to tumble rapidly over relatively smaller length scales. Therefore, for finite aspect ratio fibers, the statistical and the tensorial orientation results do not agree well due to rapid fiber tumbling near the wall. Figures $10 \mathrm{~d}$ and $12 \mathrm{~d}$ show the variation of the orientation angles across the channel width at some distance from the diverging section. After the diverging section, the infinite aspect ratio fibers near the wall tend to be rapidly aligned with the flow due to the high shear rate. At this point all the fibers in the flow domain have negative preferred orientations due to the lasting effects of the diverging geometry. Figure $10 \mathrm{~d}$ indicates a very smooth transition of the orientation angle from $-90^{\circ}$ at the centerline to $0^{\circ}$ at the wall where the agreement between all the solution techniques is excellent. In the case of finite aspect ratio fibers, due to high shear rates near the channel wall, the fourth-order tensor solution is not in good agreement with the analytical solutions in a region dominated by high shear as seen in Figures 12 and 13. However, the orientation variations are because of the rapid fiber tumbling and confined in a rather narrow band near the wall. Figures $10 \mathrm{e}$ and $12 \mathrm{e}$ illustrate the variation of the orientation angle at the midpoints of the half channel width along the channel length (i.e., $x=0-12$ ). As is seen from the graphs, there are two crossovers of the orientation angles from the negative side to the positive and back in the diverging section of the channel. The first crossover from

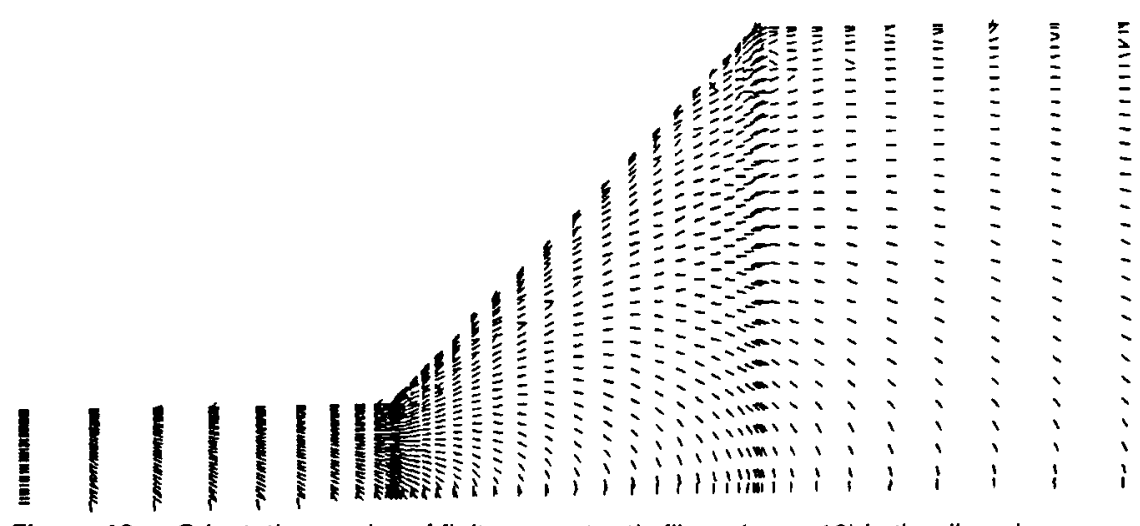

Figure 13a. Orientation angles of finite aspect ratio fibers $\left(a_{p}=10\right)$ in the diverging geometry obtained by tracing nine fibers. 


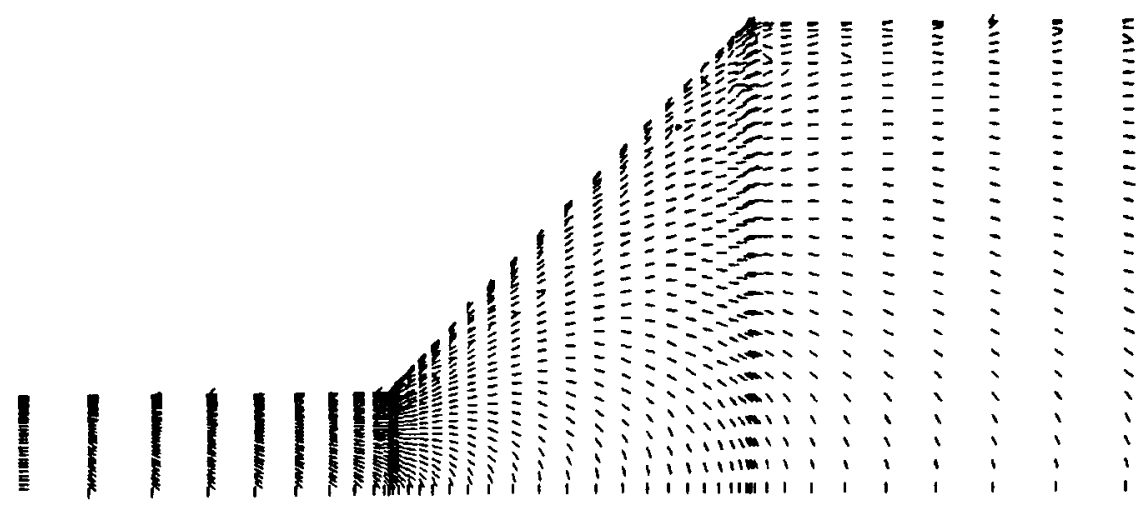

Figure 13b. Orientation angles of finite aspect ratio fibers $\left(a_{p}=10\right)$ in the diverging geometry obtained by tracing ninety fibers.

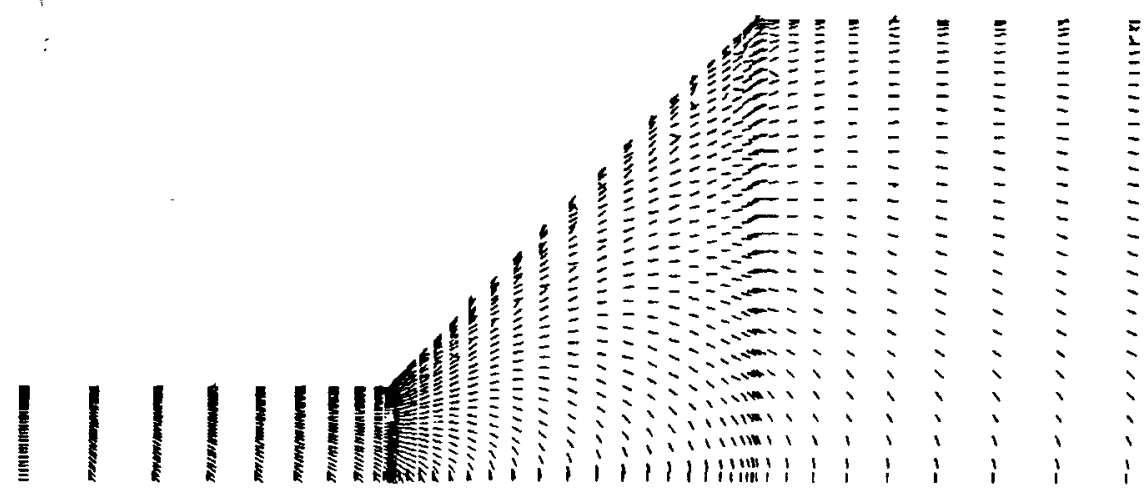

Figure 13c. Orientation angles of finite aspect ratio fibers $\left(a_{p}=10\right)$ in the diverging geometry obtained from the solution of fourth-order orientation tensor $S_{i j k}$. 
the negative 10 positive orientation could be attributed to the expanding and rapidly decelerating flow at the inlet of the diverging section, while the second crossover from the positive to negative orientation is due to the eflect of the stratight channel at the exit of the diverging section.

\section{CONCLUDING: REMARKS}

In this paper, various solution strategies are presented for the orientation field in a Newtonian fiber suspension with both infinite and finite aspect ratio fibers. Forty-five-degree converging and diverging channel geometries are considered in the analysis. In this work, the orientation field is characterized by the maximum angle of orientation which is obtained by considering second-order orientation tensors, fourth-order orientation tensors, stalistical orientation distribution by tracing nine fibers, and statistical orientation distribution by tracing ninety fibers. Random initial fiber orientation is specified at the inlet of the channels. Only maximum orientation angles are reported from all the methods considered. The orientation results obtained using the statistical orientation distribution function reveal that the accuracy of the orientation description improves with increase in the number of fibers. The solution ohtained by tracing only nine fibers gave mostly salisfictory predictions. Although increasing the number of tibers to ninety improved the accuracy considerably, one also has to consider the increase in the required computational time. Hence, the number of fibers utilized in the statistical solution can be decided based on the desired aceuracy and the available computational resources. The maximum orientation angles reported from the second-and the fourth-order orientation tensor solutions are found to be identical. Thus, it can be concluded that the results from tensorial quantities differ only in the clegree of alignment, which is represented by the relative magnitude of the eigenvalues of the second-order tensors. However, predicting correct preferred orientation does not warrant correct prediction of rheological properties of the fiber suspension if higher fiber concentrations are used. For such cases, the accuracies of these techniques in predicting the rheological properties of the anisotropic suspensions need to be investigated.

In the casc of intinite aspect ratio fibers, close agreement between the tensorial solution and the statistical solution is observed in both the geometries considered. For finite aspect ratio fibers, the tensoriat solution is in good agreement with the statistical solutions throughout most of the channel except a rather narrow band near the channel wall where rapid fiber tumbling occurs.

Due to the high shear rates existing near the channel wall, the details of the local tumbling of finite aspect ratio libers could not be accurately described. Specifically, in the regions of high velocity gradients, rapid liber tumbling takes place on a length scale much smaller than the overall flow dimensions. Therefore, capturing local fiber tumbling accuratcly with rassonable computational cffort may require some sorl of adaplive mesh generation for the orientation predictions which redistributes the nodal points considering the orientation field gradients. 


\section{APPENDIX}

The transformation of the governing equations trom the physical domain $(x, y)$ to the computational domain $(\xi, \eta)$ is performed using calculus of variables. Partial derivatives with respeed to (arlesian coxordinates are related to partial derivatives with respect to curvilinear coordinates by the chain rule of differentiation. The following results were obtained for the metrics of the transformation

$$
\xi_{2}=\frac{y_{\eta}}{J} \quad \xi_{v}=-\frac{x_{\eta}}{J} \quad \eta_{1}=-\frac{y_{\xi}}{J} \quad \eta_{v}=\frac{x_{\xi}}{J}
$$

where the Jacobian $J$ is given by

$$
J=x_{l} y_{\eta}-y_{\xi} x_{\eta}
$$

Also, using the chain rule of differentiation, the quantities $\left(\xi_{\ldots}, \eta_{1,}, \xi_{w}\right.$, etc. $)$ were found to be:

$$
\begin{aligned}
& \xi_{n}=\frac{1}{J^{2}}\left[y_{\eta !} y_{\eta}-y_{\eta}^{2} \frac{J_{l}}{J}-y_{\eta \eta} y_{5}+y_{\eta} y_{\xi} \frac{J_{\eta \eta}}{J}\right] \\
& \eta_{x x}=\frac{1}{J^{2}}\left[y_{\eta \xi} y_{\xi}-y_{\xi}^{2} \frac{J_{\eta}}{J}-y_{\eta !} \nu_{\xi \varepsilon}+y_{\eta} y_{\xi} \frac{J_{\xi}}{J}\right] \\
& \xi_{y y}=\frac{1}{J^{2}}\left[x_{\eta \varepsilon} x_{\eta}-x_{\eta}^{2} \frac{J_{\xi}}{J}-x_{\eta \eta} x_{\xi}+x_{\eta} x_{l}-\frac{J_{\eta}}{J}\right] \\
& \xi_{\eta \eta}=\frac{1}{J^{2}}\left[x_{\eta \xi} x_{\xi}+x_{\varepsilon} x_{\eta} \frac{J_{\xi}}{J}-x_{\xi \xi} x_{\eta}-x_{\xi}^{2} \frac{J_{\eta}}{J}\right] \\
& \xi_{y_{1}}=\frac{1}{J^{2}}\left[x_{\eta \eta} y_{\xi}-x_{\eta \xi} y_{\eta}+\frac{x_{\eta}}{J}\left(y_{\eta} J_{\xi}-y_{\xi} J_{\eta}\right)\right] \\
& \eta_{y x}=\frac{1}{J^{2}}\left[x_{\xi \xi} y_{\eta}-x_{\eta \xi} y_{\xi}+\frac{x_{\varepsilon}}{J}\left(y_{\xi} J_{\eta}-y_{\eta} J_{\xi}\right)\right] \\
& J_{\xi}=\left(x_{\varepsilon} y_{n \xi}+y_{n} x_{k \ell}\right)-\left(r_{n k} y_{\ell}+x_{\eta} y_{k l}\right) \\
& I_{\eta \prime}=\left(x_{\xi} y_{\eta \eta}+y_{\eta} x_{\xi \eta}\right)-\left(x_{\eta \eta} y_{\xi}+x_{\eta} y_{\xi \eta}\right)
\end{aligned}
$$

All the above metric quantilies are calculated numerically using central differencing. One sided difference relations are used for the boundaries. In all the difference relations, $\Delta \xi$ and $\Delta \eta$ are taken to be equal to unity. 


\section{REFERENCES}

1. Jeffery, G. B. 1922. "The Motion of Ellipsoidal Particles Immersed in a Viscous Fluid," Proc. Roy. Soc., 102A:161.

2. Hinch, E. J. and L. G. Leal. 1975. "Constitutive Equations in Suspension Mechanics. Part I. General Formulation," J. Fluid Mech., 71:481.

3. Evans, J. G. 1975. "The Flow of Suspension of Force-Free Rigid Rods in a Newtonian Fluid," Ph.D. dissertation, Cambridge University.

4. Dinh, S. M. and R. C. Armstrong. 1984. "A Rheological Equation State for Semiconcentrated Fiber Suspensions," J. Rheol., 28:207.

5. Lipscomb, G. G., M. M. Denn, D. U. Hur and D. V. Boger. 1988. "Flow of Fiber Suspensions in Compex Geometries," J. Non-Newtonian Fluid Mech., 26:297.

6. Phan-Thien, N. and A. L. Graham. 1991. "A New Constitutive Model for Fibre Suspensions: Flow Past a Sphere," Rheol. Acta, 30:44.

7. Tucker, C. L. 1991. "Flow Regimes for Fiber Suspensions in Narrow Gaps," J. Non-Newtonian Fluid Mech., 39:239.

8. Givler, R. C., M. J. Crochett and R. B. Pipes. 1983. "Numerical Prediction of Fiber Orientations in Dilute Suspensions," J. Comp. Matl., 17:330.

9. Papanastasiou, T. C. and A. N. Alexandrou. 1987. "Isothermal Extrusion of Non-Dilute Fiber Suspensions," J. Non-Newtonian Fluid Mech., 25:995.

10. Chiba, K. and K. Nakamura. 1990. "A Numerical Solution for the Flow of Dilute Fiber Suspensions through an Axisymmetric Contraction," J. Non-Newtonian Fluid Mech., 35:1.

11. Rosenberg, J., M. Denn and R. Keunings. 1990. "Simulation of Non-Recirculating Flows of Dilute Fiber Suspensions," J. Non-Newtonian Fluid Mech., 37:317.

12. Altan, M. C., S. I. Güçeri and R. B. Pipes. 1991. In print. "Anisotropic Channel Flow of Fiber Suspensions," J. Non-Newtonian Fluid Mech.

13. Goldsmith, H. L. and S. G. Mason. 1967. Rheology: Theory and Applications, Academic Press, p. 85 .

14. Vincent, M. and J. F. Agassant. 1985. "Experimental and Theoretical Study of Short Fiber Orientation in Diverging Flows," Rheol. Acta., 24:603.

15. Akbar, S. and M. C. Altan. 1991. In print. "On the Solution of Fiber Orientation in TwoDimensional Homogeneous Flows," Poly. Eng. Sci.

16. Altan, M. C., S. G. Advani, S. I. Güçeri and R. B. Pipes. 1989. "On the Description of the Orientation State for Fiber Suspensions in Homogeneous Flows," J. Rheol, 33:1129.

17. Advani, S. G. and C. L. Tucker. 1987. "The Use of Tensors to Describe and Predict Fiber Orientation in Short Fiber Composites," J. Rheol., 31:751.

18. Thompson, J. F., Z. U. A. Warsi and C. W. Mastin. 1985. Numerical Grid Generation. New York: Elsevier Science. 